\title{
Poisson and Hochschild cohomology and the semiclassical limit
}

\author{
Matthew Towers
}

\begin{abstract}
Let $\mathrm{A}$ be a quantum algebra possessing a semiclassical limit $A$. We show that under certain hypotheses $A^{e}$ can be thought of as a deformation of the Poisson enveloping algebra of $A$, and we give a criterion for the Hochschild cohomology of $\mathrm{A}$ to be a deformation of the Poisson cohomology of $A$ in the case that A is Koszul. We verify that condition for the algebra of $2 \times 2$ quantum matrices and calculate its Hochschild cohomology and the Poisson cohomology of its semiclassical limit.
\end{abstract}

Mathematics Subject Classification (2010). 16E40, 17B63, 16S37.

Keywords. Poisson cohomology, Hochschild cohomology, semiclassical limit, Koszul algebra, deformations.

\section{Introduction}

Certain quantum algebras A admit a semiclassical limit: that is, a Poisson algebra structure on their $q \rightarrow 1$ limit $A$ which preserves some noncommutative information from A. A simple example is the quantum plane

$$
\mathrm{A}=\frac{k(q)\langle\mathrm{x}, \mathrm{y}\rangle}{(\mathrm{xy}-q \mathrm{yx})}
$$

whose semiclassical limit is the polynomial ring $k[x, y]$ with Poisson structure determined by $\{x, y\}=x y$. Various authors have investigated to what extent the Hochschild (co)homology of $A$ is determined by the Poisson (co)homology of its semiclassical limit, for example [16, 5], usually using spectral sequence methods, and a related situation where there is a Poisson structure on an associated graded algebra is dealt with in [9, Théorème, p.223]. The famous Kontsevich quantization theorem also guarantees a relationship between the cohomology of a Poisson algebra and that of its canonical quantization.

In this paper we study the link between Poisson and Hochschild cohomology by showing that if $\mathrm{A}$ is graded with a PBW basis of polynomial type with a polynomial semiclassical limit $A$, the enveloping algebra $\mathrm{A}^{e}$ is a deformation of the Poisson enveloping algebra $P(A)$ (Section 3). The restriction to $A$ being polynomial allows 
us to describe $P(A)$ by generators and relations (Lemma 2.2): when the Kähler differentials of $A$ are not free as an $A$-module, additional relations are required.

If $A$ is Koszul with quadratic dual $A !$ the Hochschild cohomology of $A$ is computed by a differential graded algebra whose underlying algebra is $A \otimes A !$. If A has a PBW basis of polynomial type we show that this DGA is a deformation of the DGA $A \otimes A^{!}$that computes Poisson cohomology of the semiclassical limit $A$ (Proposition 4.10). This leads to a condition for $\mathrm{HH}(\mathrm{A})$ to be a deformation of $\mathrm{HP}(A)$; in particular when this holds the Hochschild and Poisson cohomologies have the same bigraded Hilbert series (Corollary 4.11).

Two algebras to which our results can be applied are the coordinate ring $\mathrm{A}(n)$ of quantum affine $n$-space and the algebra $\mathrm{M}$ of $2 \times 2$ quantum matrices. We discuss the cohomology of the quantum plane and its semiclassical limit in Section 5 as an example of our methods; Hochschild cohomology for quantum affine spaces and Poisson cohomology of their semiclassical limits are already known, for example [17, §6], [1], [11, Proposition 2.2.1], [15, §3.3].

In Section 6 we show that the Hochschild cohomology for $2 \times 2$ quantum matrices is a $q$-deformation of the Poisson cohomology of the semiclassical limit, and calculate this Poisson cohomology explicitly in Theorem 6.1. These computations are new, although some low-dimensional Hochschild cohomology groups for quantum matrices have appeared in the literature: the zeroth Hochschild cohomology group is known to be generated by the quantum determinant, and the structure of the first cohomology group as a module over the centre was determined in [10]. An interesting feature of these computations is the action of the Poisson centre on the Poisson cohomology groups, which acts freely except for a single trivial summand in the top cohomological dimension. This is in contrast to [16, Theorem 4.1] for example, where the action is free.

1.1. Notation and conventions. Throughout this article $q$ is a transcendental element over a field $k$ of characteristic zero. $k\left[q^{ \pm 1}\right]$ is the ring of Laurent polynomials in $q$ and $k(q)$ its field of fractions. "Graded" means $\mathbb{Z}_{\geq 0}$-graded unless otherwise stated. The $n$th graded component of a graded algebra $\Lambda$ is denoted $\Lambda_{n}$, and if $\lambda \in \Lambda_{n}$ we write $|\lambda|=n$.

\section{Poisson algebras and Poisson cohomology}

2.1. The Poisson enveloping algebra. Let $A$ be a Poisson $k$-algebra with bracket $\{-,-\}$; that is, $A$ is a commutative associative $k$-algebra with unit, $\{-,-\}$ is a Lie bracket on $A$, and for any $a \in A$ the map

$$
b \mapsto\{a, b\}
$$

is a derivation. A left Poisson module over $A$ is a $k$-vector space $M$ which is a simultaneously a left module for $A$ as an associative algebra and a left module for 
the Lie algebra $(A,\{-.-\})$, satisfying

$$
\begin{aligned}
& \{x, y m\}_{M}=\{x, y\} m+y\{x, m\}_{M} \\
& \{x y, m\}_{M}=x\{y, m\}_{M}+y\{x, m\}_{M}
\end{aligned}
$$

for any $x, y \in A$ and $m \in M$, where $\{a, m\}_{M}$ denotes the Lie algebra action and $a m$ the associative algebra action of $a \in A$ on $m \in M$.

Let $\Omega(A)$ be the $A$-module of Kähler differentials of $A$. This is the free left $A$ module on generators $\Omega(a)$ for $a \in A$ quotiented by the submodule generated by all elements of the form

$$
\Omega(\alpha), \quad \Omega(a+b)-\Omega(a)-\Omega(b), \quad \Omega(a b)-a \Omega(b)-b \Omega(a)
$$

for $\alpha \in k$ and $a, b \in A$. If we define

$$
[a \Omega(x), b \Omega(y)]=a\{x, b\} \Omega(y)+b\{a, y\} \Omega(x)+a b \Omega(\{x, y\})
$$

then $\Omega(A)$ becomes a $k$-Lie algebra: see [8, Theorem 3.8].

There is an associative algebra $U(A, \Omega(A))$ called the Poisson enveloping algebra satisfying a universal property such that the category of left $U(A, \Omega(A))$ modules is equivalent to the category of left Poisson modules over $A$. A more general construction of which the Poisson enveloping algebra is a special case given in $[8$, $\S 1]$.

In the rest of this section $A=k\left[x_{1}, \ldots, x_{n}\right]$ will be a polynomial algebra so that the Kähler differentials are freely generated as an $A$-module by $\Omega\left(x_{1}\right), \ldots, \Omega\left(x_{n}\right)$ $[18, \S 8.8]$. We will need a presentation of the Poisson enveloping algebra by generators and relations in this special case. To this end, let $P(A)$ be the $k$-algebra generated by $y_{i}$ and $\Omega\left(y_{i}\right)$ for $1 \leq i \leq n$, subject to two sets of relations. The first set says that the $y_{i}$ s commute:

$$
y_{i} y_{j}=y_{j} y_{i} \quad 1 \leq i<j \leq n
$$

so there is an algebra homomorphism $\iota_{A}: A \rightarrow P(A)$ determined by $x_{i} \mapsto y_{i}$. The second set of relations is

$$
\begin{gathered}
\Omega\left(y_{i}\right) y_{j}-y_{j} \Omega\left(y_{i}\right)=\iota_{A}\left(\left\{x_{i}, x_{j}\right\}\right) \quad 1 \leq i, j \leq n \\
\Omega\left(y_{i}\right) \Omega\left(y_{j}\right)-\Omega\left(y_{j}\right) \Omega\left(y_{i}\right)=\sum_{k} \iota_{A}\left(\frac{\partial\left\{x_{i}, x_{j}\right\}}{\partial x_{k}}\right) \Omega\left(y_{k}\right) \quad 1 \leq i<j \leq n .
\end{gathered}
$$

The map $\iota_{A}: A \rightarrow P(A)$ makes $P(A)$ into an $A$-module. Let $\iota_{\Omega(A)}: \Omega(A) \rightarrow P(A)$ be the map of $A$-modules such that $\Omega\left(x_{i}\right) \mapsto \Omega\left(y_{i}\right)$, and regard $P(A)$ as a $k$-Lie algebra by defining $[u, v]=u v-v u$. 
Lemma 2.1. $\iota_{\Omega(A)}$ is a homomorphism of $k$-Lie algebras.

Proof. Firstly note that

$$
\Omega\left(y_{i}\right) \iota_{A}(b)-\iota_{A}(b) \Omega\left(y_{i}\right)=\iota_{A}\left(\left\{x_{i}, b\right\}\right)
$$

for any $b \in A$. When $b$ is a monomial this can be proved by induction on the length using (2.3), and the result extends to arbitrary $b \in A$ by linearity.

To prove that $\iota_{\Omega(A)}$ is a Lie algebra homomorphism we must show that for any $a, b \in A$ and $1 \leq i, j \leq n$,

$$
\begin{aligned}
\iota_{\Omega(A)}\left(\left[a \Omega\left(x_{i}\right), b \Omega\left(x_{j}\right)\right]\right)=\iota_{A}\left(a\left\{x_{i}, b\right\}\right) \Omega\left(y_{j}\right)+\iota_{A}\left(b\left\{a, x_{j}\right\}\right) \Omega\left(y_{i}\right) \\
+\iota_{A}(a b) \sum_{k} \iota_{A}\left(\frac{\partial\left\{x_{i}, x_{j}\right\}}{\partial x_{k}}\right) \Omega\left(y_{k}\right)
\end{aligned}
$$

is equal to

$$
\begin{aligned}
& \iota_{\Omega(A)}\left(a \Omega\left(x_{i}\right)\right) \iota_{\Omega(A)}\left(b \Omega\left(x_{j}\right)\right)-\iota_{\Omega(A)}\left(b \Omega\left(x_{j}\right)\right) \iota_{\Omega(A)}\left(a \Omega\left(x_{i}\right)\right) \\
& =\iota_{A}(a) \Omega\left(y_{i}\right) \iota_{A}(b) \Omega\left(y_{j}\right)-\iota_{A}(b) \Omega\left(y_{j}\right) \iota_{A}(a) \Omega\left(y_{i}\right)
\end{aligned}
$$

for any $a, b \in A$ and any $i, j$.

Using (2.5),

$$
\begin{aligned}
\iota_{A}(a) \Omega\left(y_{i}\right) \iota_{A}(b) \Omega\left(y_{j}\right)-\iota_{A}(b) \Omega\left(y_{j}\right) \iota_{A}(a) \Omega\left(y_{i}\right) \\
=\iota_{A}(a)\left(\iota_{A}(b) \Omega\left(y_{i}\right)+\iota_{A}\left(\left\{x_{i}, b\right\}\right)\right) \Omega\left(y_{j}\right) \\
\quad-\iota_{A}(b)\left(\iota_{A}(a) \Omega\left(y_{j}\right)+\iota_{A}\left(\left\{x_{j}, a\right\}\right)\right) \Omega\left(y_{i}\right) \\
=\iota_{A}(a b)\left(\Omega\left(y_{i}\right) \Omega\left(y_{j}\right)-\Omega\left(y_{j}\right) \Omega\left(y_{i}\right)\right) \\
\quad+\iota_{A}\left(a\left\{x_{i}, b\right\}\right) \Omega\left(y_{j}\right)+\iota_{A}\left(b\left\{a, x_{j}\right\}\right) \Omega\left(y_{i}\right)
\end{aligned}
$$

and the result follows from (2.4)

Lemma 2.2. The Poisson enveloping algebra $U(A, \Omega(A))$ is isomorphic to $P(A)$.

Proof. We will verify that $\left(P(A), \iota_{A}, \iota_{\Omega(A)}\right)$ has the universal property of $[8,1.6]$. We must show that given an associative $k$-algebra $B$ equipped with the Lie bracket $\left[b_{1}, b_{2}\right]=b_{1} b_{2}-b_{2} b_{1}$, a morphism of $k$-Lie algebras $\phi_{\Omega(A)}: \Omega(A) \rightarrow B$ and a morphism of $k$-algebras $\phi_{A}: A \rightarrow B$ such that

$$
\begin{aligned}
\phi_{A}(a) \phi_{\Omega(A)}\left(b \Omega\left(x_{i}\right)\right) & =\phi_{\Omega(A)}\left(a b \Omega\left(x_{i}\right)\right) \\
\phi_{\Omega(A)}\left(b \Omega\left(x_{i}\right)\right) \phi_{A}(a)-\phi_{A}(a) \phi_{\Omega(A)}\left(b \Omega\left(x_{i}\right)\right) & =\phi_{A}\left(b\left\{x_{i}, a\right\}\right)
\end{aligned}
$$

for any $a, b \in A$ and any $i$, there is a unique homomorphism $\Phi: P(A) \rightarrow B$ of $k$-algebras such that $\Phi \circ \iota_{A}=\phi_{A}$ and $\Phi \circ \iota_{\Omega(A)}=\phi_{\Omega(A)}$. 
If these are to hold, $\Phi$ must satisfy $\Phi\left(y_{i}\right)=\phi_{A}\left(x_{i}\right)$ and $\Phi\left(\Omega\left(y_{i}\right)\right)=$ $\phi_{\Omega(A)}\left(\Omega\left(x_{i}\right)\right)$. Since the elements $y_{i}$ and $\Omega\left(y_{i}\right)$ generate $P(A)$, if such a $\Phi$ exists it is unique.

In order to show that such a $\Phi$ exists we need only show that the elements $\phi_{A}\left(x_{i}\right)$ and $\phi_{\Omega(A)}\left(\Omega\left(x_{i}\right)\right)$ satisfy the relations (2.2), (2.3) and (2.4) of $P(A)$. Certainly the $\phi_{A}\left(x_{i}\right)$ commute since $\phi_{A}: A \rightarrow B$ is an algebra homomorphism, so (2.2) is satisfied. Next,

$$
\phi_{\Omega(A)}\left(\Omega\left(x_{i}\right)\right) \phi_{A}\left(x_{j}\right)-\phi_{A}\left(x_{j}\right) \phi_{\Omega(A)}\left(\Omega\left(x_{i}\right)\right)=\phi_{A}\left(\left\{x_{i}, x_{j}\right\}\right)
$$

by (2.7), and therefore (2.3) is satisfied. Finally,

$$
\begin{aligned}
\phi_{\Omega(A)}\left(\Omega\left(x_{i}\right)\right) \phi_{\Omega(A)}\left(\Omega\left(x_{j}\right)\right)-\phi_{\Omega(A)}\left(\Omega\left(x_{j}\right)\right) & \phi_{\Omega(A)}\left(\Omega\left(x_{i}\right)\right) \\
& =\phi_{\Omega(A)}\left(\left[\Omega\left(x_{i}\right), \Omega\left(x_{j}\right)\right]\right) \\
& =\phi_{\Omega(A)}\left(\Omega\left(\left\{x_{i}, x_{j}\right\}\right)\right.
\end{aligned}
$$

because $\phi_{\Omega(A)}$ is a homomorphism of Lie algebras, and applying $\phi_{\Omega(A)}$ to

$$
\Omega\left(\left\{x_{i}, x_{j}\right\}\right)=\sum_{k} \frac{\partial\left\{x_{i}, x_{j}\right\}}{\partial x_{k}} \Omega\left(x_{k}\right)
$$

and using (2.6) gives

$$
\phi_{\Omega(A)}\left(\Omega\left(\left\{x_{i}, x_{j}\right\}\right)\right)=\sum_{k} \phi_{A}\left(\frac{\partial\left\{x_{i}, x_{j}\right\}}{\partial x_{k}}\right) \phi_{\Omega(A)}\left(\Omega\left(x_{k}\right)\right)
$$

and so (2.4) is also satisfied.

Corollary 2.3. $P(A)$ has a $P B W$ basis consisting of all elements of the form

$$
y_{1}^{a_{1}} \cdots y_{n}^{a_{n}} \Omega\left(y_{1}\right)^{b_{1}} \cdots \Omega\left(y_{n}\right)^{b_{n}}
$$

for $a_{i}, b_{i} \geq 0$.

Proof. This is a consequence of a result of Rinehart [8, Theorem 1.9] which shows that the Poisson enveloping algebra is isomorphic as a vector space to the symmetric $A$-algebra on $\Omega(A)$ via the canonical map.

2.2. Poisson cohomology. In this section $A=k\left[x_{1}, \ldots, x_{n}\right]$ is once again a polynomial algebra so that $\Omega(A)$ is a free $A$-module, and we may consider its $m$ th $A$-exterior power $\Lambda_{A}^{m}(\Omega(A))$. This is the quotient of the tensor product over $A$ of $m$ copies of $\Omega(A)$ by the submodule generated by all tensors with two equal factors. 
Write

$$
\Omega\left(a_{1}\right) \wedge \cdots \wedge \Omega\left(a_{m}\right)
$$

for the image of the pure tensor

$$
\Omega\left(a_{1}\right) \otimes_{A} \cdots \otimes_{A} \Omega\left(a_{m}\right)
$$

in $\bigwedge_{A}^{m}(\Omega(A))$. As an $A$-module, $\bigwedge_{A}^{m}(\Omega(A))$ is free on all elements of the form

$$
\Omega\left(x_{i_{1}}\right) \wedge \cdots \wedge \Omega\left(x_{i_{m}}\right)
$$

for $1 \leq i_{1}<i_{2}<\cdots<i_{m} \leq n$, so it is isomorphic to $A \otimes_{k} \Lambda^{m}(V)$ where $\Lambda^{m}(V)$ is the $m$ th exterior power of the vector space $V$ spanned by the $\Omega\left(x_{i}\right)$.

Definition 2.4. $\operatorname{Alt}_{A}^{m}(\Omega(A), A)$ is $\operatorname{Hom}_{A}\left(\bigwedge_{A}^{m}(\Omega(A)), A\right)$

This is what Huebschmann calls the space of $A$-multilinear alternating functions from $\Omega(A)$ to $A$. The direct sum $\operatorname{Alt}_{A}^{*}(\Omega(A), A)=\bigoplus_{m \geq 0} \operatorname{Alt}_{A}^{m}(\Omega(A), A)$ is a differential graded algebra when equipped with the Cartan-Chevalley-Eilenberg differential

$$
\begin{aligned}
d f\left(\Omega\left(x_{i_{1}}\right) \wedge\right. & \left.\cdots \wedge \Omega\left(x_{i_{m}}\right)\right) \\
=\sum_{j \geq 1}( & -1)^{j+1} \Omega\left(x_{i_{j}}\right) f\left(\Omega\left(x_{i_{1}}\right) \wedge \cdots \wedge \widehat{\Omega\left(x_{i_{j}}\right)} \wedge \cdots \wedge \Omega\left(x_{i_{m}}\right)\right) \\
\quad+\sum_{1 \leq j<k \leq m}(-1)^{j+k} f\left(\left[\Omega\left(x_{i_{j}}\right), \Omega\left(x_{i_{k}}\right)\right] \wedge \Omega\left(x_{i_{1}}\right) \wedge \cdots\right. & \\
& \left.\wedge \widehat{\Omega\left(x_{i_{j}}\right)} \wedge \cdots \wedge \widehat{\Omega\left(x_{i_{k}}\right)} \wedge \cdots \wedge \Omega\left(x_{i_{m}}\right)\right)
\end{aligned}
$$

and the shuffle product

$$
\begin{aligned}
& (f \wedge g)\left(\Omega\left(a_{1}\right) \wedge \cdots \wedge \Omega\left(a_{|f|+|g|}\right)\right) \\
& \quad=\sum_{\mathbf{i}} \operatorname{sgn}(\mathbf{i}) f\left(\Omega\left(a_{i_{1}}\right) \wedge \cdots \wedge \Omega\left(a_{i_{|f|} \mid}\right) g\left(\Omega\left(a_{i_{|f|+1}}\right) \wedge \cdots \wedge \Omega\left(a_{i_{|f|+|g|}}\right)\right)\right.
\end{aligned}
$$

where $f \in \operatorname{Alt}_{A}^{|f|}(\Omega(A), A), g \in \operatorname{Alt}_{A}^{|g|}(\Omega(A), A)$, the sum is over all $\mathbf{i}=$ $\left(i_{1}, \ldots, i_{|f|+|g|}\right)$ such that $i_{1}<\cdots<i_{|f|}$ and $i_{|f|+1}<\cdots<i_{|f|+|g|}$, and $\operatorname{sgn}(\mathbf{i})$ is the sign of the permutation $r \mapsto i_{r}$. The shuffle product on the space of alternating forms is obtained by transferring the natural multiplication on $A \otimes_{k} \Lambda^{*}\left(V^{*}\right)$ through the isomorphisms

$$
\operatorname{Alt}_{A}(\Omega(A), A) \cong \operatorname{Hom}_{A}\left(A \otimes \Lambda^{*}(V), A\right) \cong \operatorname{Hom}_{k}\left(\Lambda^{*}(V), A\right) \cong A \otimes_{k} \Lambda^{*}\left(V^{*}\right) .
$$

Definition 2.5. The Poisson cohomology $\operatorname{HP}^{*}(A)$ of a Poisson algebra $A$ is the cohomology of the differential graded algebra $\operatorname{Alt}_{A}^{*}(\Omega(A), A)$. 
If $A$ is a graded algebra and its Poisson bracket respects the grading then the Poisson cohomology groups are bigraded; in this case we write $\operatorname{HP}^{i j}(A)$ for the part in homological degree $i$ and internal degree $j$.

When the Kähler differentials are projective as an $A$-module, so in particular when $A$ is polynomial, $P(A) \otimes_{A} \Lambda_{A}^{*}(\Omega(A))$ is a projective resolution of $A$ as a $P(A)$-module and the Poisson cohomology of $A$ is isomorphic as an algebra to $\operatorname{Ext}_{P(A)}^{*}(A, A)[8, \mathrm{p} .81]$.

\section{The semiclassical limit and $q$-deformations}

Let $\mathcal{A}$ be a $k\left[q^{ \pm 1}\right]$-algebra which is a torsion-free $k\left[q^{ \pm 1}\right]$-module and suppose $A=$ $\mathcal{A} /(q-1) \mathcal{A}$ is commutative. If $u \in \mathcal{A}$ write $\bar{u}$ for its image in $A$. Then $A$ is a Poisson algebra with bracket

$$
\{\bar{a}, \bar{b}\}:=\overline{\beta(a, b)}
$$

where $\beta(a, b)$ is the unique element of $\mathcal{A}$ such that $a b-b a=(q-1) \beta(a, b)$.

Definition 3.1. With $\mathcal{A},\{-,-\}$ and $A$ as above we say that $A$ is the semiclassical limit of $\mathcal{A}$.

See for example $[4,7]$.

Definition 3.2. A $k\left[q^{ \pm 1}\right]$-subalgebra $\mathcal{R}$ of a $k(q)$-algebra $\mathrm{R}$ is called a $k\left[q^{ \pm 1}\right]$-form of $\mathrm{R}$ if the natural map $\mathcal{R} \otimes_{k\left[q^{ \pm 1}\right]} k(q) \rightarrow \mathrm{R}$ is an isomorphism. We say $\mathrm{R}$ is a $q$ deformation of the $k$-algebra $R$ via the $k\left[q^{ \pm 1}\right]$-form $\mathcal{R}$ if $\mathcal{R} /(q-1) \mathcal{R}$ is isomorphic as a $k$-algebra to $R$.

We will use an analogous notion of $q$-deformation for differential graded algebras, obtained by replacing 'algebra' and 'subalgebra' by 'DG-algebra' and 'sub-DG-algebra' everywhere in the above definition. Note that if $\mathrm{R}$ is (bi)graded then so is $R$, and they have the same Hilbert series.

Suppose $\mathcal{A}$ is a $k\left[q^{ \pm 1}\right]$-form of a $k(q)$-algebra $\mathrm{A}$ and that $\mathcal{A}$ has a semiclassical limit $A$. We want to relate the Poisson enveloping algebra $P(A)$ and the enveloping algebra $\mathrm{A}^{e}=\mathrm{A} \otimes_{k(q)} \mathrm{A}^{\mathrm{op}}$ of $\mathrm{A}$ using the notion of $q$-deformation. The subalgebra of $\mathrm{A}^{e}$ generated by $\mathcal{A} \otimes 1$ and $1 \otimes \mathcal{A}^{\mathrm{op}}$ is not suitable as a $k\left[q^{ \pm 1}\right]$-form because it is commutative modulo $q-1$, and $P(A)$ is in general noncommutative. To help us define a suitable $k\left[q^{ \pm 1}\right]$-form we need some special elements of $\mathrm{A}^{e}$.

Definition 3.3. Let $a \in A$. Then $\tilde{\Omega}(a)$ is

$$
\frac{\mathrm{a} \otimes 1-1 \otimes \mathrm{a}}{q-1} \in \mathrm{A}^{e} .
$$


Lemma 3.4. Let $x, y \in \mathcal{A}$. Write $\mathrm{x}$ and $\mathrm{y}$ for the elements $x \otimes 1$ and $y \otimes 1$ of $\mathrm{A}^{e}$. Then in $\mathrm{A}^{e}$,

$$
\begin{aligned}
\tilde{\Omega}(x y) & =\mathrm{x} \tilde{\Omega}(y)+\mathrm{y} \tilde{\Omega}(x)+(q-1) \tilde{\Omega}(y) \tilde{\Omega}(x) \\
\tilde{\Omega}(x) \mathrm{y}-\mathrm{y} \tilde{\Omega}(x) & =\beta(x, y) \otimes 1 \\
\tilde{\Omega}(x) \tilde{\Omega}(y)-\tilde{\Omega}(x) \tilde{\Omega}(y) & =\tilde{\Omega}(\beta(x, y)) .
\end{aligned}
$$

The proof is a simple computation. This lemma shows that the $\tilde{\Omega}(\mathrm{a})$ behave like $q$-analogues of the Kähler differentials of $A$ - compare (3.1) with (2.1) and (3.2), (3.3) with (2.3), (2.4).

Remark 3.5. If $A$ is a quadratic algebra generated by homogeneous elements $x_{i}$ of degree one with a basis of the form $x_{1}^{a_{1}} \cdots x_{n}^{a_{n}}$ for $a_{i} \geq 0$, these relations, together with the relations of $A$, are enough to give a presentation of $A^{e}$. This follows by counting the dimension of the spaces of relations.

For the rest of this section we assume that $\mathrm{A}$ is graded, that $\mathrm{x}_{1}, \ldots, \mathrm{x}_{n}$ is a homogeneous generating set for $\mathrm{A}$, and that $A$ is polynomial on the images $\overline{\mathrm{x}_{i}}$ of the $\mathrm{x}_{i}$. Let $y_{i}$ be the generator of $P(A)$ corresponding to $\overline{\mathrm{x}_{i}}$; then $P(A)$ is graded by putting $y_{i}$ in the same degree as $\mathrm{x}_{i}$.

Lemma 3.6. Let $\mathcal{A}^{\prime}$ be the $k\left[q^{ \pm 1}\right]$-subalgebra of $\mathrm{A}^{e}$ generated by $\mathrm{x}_{i} \otimes 1$ and $\tilde{\Omega}\left(\mathrm{x}_{i}\right)$ for $1 \leq i \leq n$. Then there is a surjection of graded algebras $P(A) \rightarrow \mathcal{A}^{\prime} /(q-1) \mathcal{A}^{\prime}$ defined by $y_{i} \mapsto \overline{\mathrm{x}_{i} \otimes 1}$ and $\Omega\left(y_{i}\right) \mapsto \overline{\tilde{\Omega}\left(\mathrm{x}_{i}\right)}$.

Proof. We must show that if we substitute $x_{i} \otimes 1$ for $y_{i}$ and $\tilde{\Omega}\left(\mathrm{x}_{i}\right)$ for $\Omega\left(y_{i}\right)$ in the defining relations (2.2), (2.3), (2.4) of $P(A)$, the resulting expressions lie in $(q-1) \mathcal{A}^{\prime}$. This is true of the relations (2.2) because the semiclassical limit exists.

(2.3): We need

$$
\tilde{\Omega}\left(\mathrm{x}_{i}\right)\left(\mathrm{x}_{j} \otimes 1\right)-\left(\mathrm{x}_{j} \otimes 1\right) \tilde{\Omega}\left(\mathrm{x}_{i}\right)=\beta\left(\mathrm{x}_{i}, \mathrm{x}_{j}\right) \otimes 1 \bmod (q-1)
$$

but this is immediate from (3.2).

(2.4): Suppose first that $\beta\left(\mathrm{x}_{i}, \mathrm{x}_{j}\right)=x_{i_{1}} \cdots x_{i_{N}}$ is a monomial. We need

$$
\tilde{\Omega}\left(\mathrm{x}_{i}\right) \tilde{\Omega}\left(\mathrm{x}_{j}\right)-\tilde{\Omega}\left(\mathrm{x}_{j}\right) \tilde{\Omega}\left(\mathrm{x}_{i}\right)=\sum_{j}\left(\mathrm{x}_{i_{1}} \cdots \widehat{\mathrm{x}_{i_{j}}} \cdots \mathrm{x}_{i_{N}} \otimes 1\right) \tilde{\Omega}\left(\mathrm{x}_{i_{j}}\right) \quad \bmod (q-1)
$$

but this follows easily by induction on $N$ using (3.1). The general case, when $\beta\left(\mathrm{x}_{i}, \mathrm{x}_{j}\right)$ is not assumed to be a monomial, follows by linearity.

Lemma 3.7. Let $\mathrm{z}_{1}, \ldots, \mathrm{z}_{n}$ be a homogeneous generating set for a graded $k(q)$ algebra $\mathrm{B}$ and let $\mathcal{B}$ be the $k\left[q^{ \pm 1}\right]$-subalgebra of $\mathrm{B}$ generated by $\mathrm{z}_{1}, \ldots, \mathrm{z}_{n}$. Then $\mathcal{B} \otimes_{k\left[q^{ \pm 1}\right]} k(q) \cong \mathrm{B}$ as graded algebras. 
Proof. The assignment $z_{i} \mapsto z_{i} \otimes 1$ determines a graded surjective homomorphism of algebras $\mathrm{B} \rightarrow \mathcal{B} \otimes_{k\left[q^{ \pm 1}\right]} k(q)$. Suppose the kernel contains a non-zero element $X=\sum_{\mathbf{i}}\left(a_{\mathbf{i}} / b_{\mathbf{i}}\right) z_{\mathbf{i}}$ where $a_{\mathbf{i}}, b_{\mathbf{i}} \in k\left[q^{ \pm 1}\right]$ and $\mathrm{z}_{\mathbf{i}}=z_{i_{1}}, \ldots, z_{i_{N}}$. We may assume all the $b_{\mathbf{i}}$ are equal to 1 by multiplying by an appropriate element of $k\left[q^{ \pm 1}\right]$, so that $X \in \mathcal{B}$. Now $\mathcal{B}$ is a free $k\left[q^{ \pm 1}\right]$-module as it is the direct sum of its graded pieces which are finitely generated $k\left[q^{ \pm 1}\right]$-submodules of a $k(q)$-module and therefore torsion-free. Therefore the map $\mathcal{B} \rightarrow \mathcal{B} \otimes_{k\left[q^{ \pm 1}\right]} k(q)$ is injective and so $X=0$, a contradiction.

It follows that $\mathcal{A}^{\prime}$ is a $k\left[q^{ \pm 1}\right]$-form of $\mathrm{A}^{e}$.

Corollary 3.8. If $\operatorname{dim}_{k} P(A)_{m}=\operatorname{dim}_{k(q)} \mathrm{A}_{m}^{e}$ for all $m$ then $\mathrm{A}^{e}$ is a q-deformation of $P(A)$ via the $k\left[q^{ \pm 1}\right]$-form $\mathcal{A}^{\prime}$.

Proof. If we regard $k$ as a $k\left[q^{ \pm 1}\right]$-module with $q$ acting as 1 then

$$
\mathcal{A}^{\prime} /(q-1) \mathcal{A}^{\prime} \cong \mathcal{A}^{\prime} \otimes_{k\left[q^{ \pm 1}\right]} k
$$

as graded algebras. Each graded piece of $\mathcal{A}^{\prime}$ is a free $k\left[q^{ \pm 1}\right]$-module, since it embeds into a $k(q)$-module $\mathrm{A}^{e}$ and is therefore torsion free. So for any $m$,

$\operatorname{dim}_{k}\left(\mathcal{A}^{\prime} \otimes_{k\left[q^{ \pm 1}\right]} k\right)_{m}=\operatorname{dim}_{k(q)}\left(\mathcal{A}^{\prime} \otimes_{k\left[q^{ \pm 1}\right]} k(q)\right)_{m}=\operatorname{dim}_{k(q)} \mathrm{A}_{m}^{e}=\operatorname{dim}_{k} P(A)_{m}$ where the second equality is because of Lemma 3.7. So the surjection of Lemma 3.6 has to be injective.

When A has a PBW basis of polynomial type we can apply the previous corollary to get:

Theorem 3.9. Suppose the set of elements of the form $x_{1}^{a_{1}} \cdots x_{n}^{a_{n}}$ for $a_{i} \geq 0$ form a basis of $\mathrm{A}$. Then $\mathrm{A}^{e}$ is a q-deformation of $P(A)$ via the $k\left[q^{ \pm 1}\right]$-form $\mathcal{A}^{\prime}$.

Proof. Corollary 2.3 says that $P(A)$ has a PBW basis consisting of the elements

$$
y_{1}^{a_{1}} \cdots y_{n}^{a_{n}} \Omega\left(y_{1}\right)^{b_{1}} \cdots \Omega\left(y_{n}\right)^{b_{n}}
$$

for $a_{i}, b_{i} \geq 0$, where $y_{i}$ is the generator of $P(A)$ corresponding to $\overline{x_{i}}$. Our assumptions on $\mathrm{A}$ mean that $\mathrm{A}^{e}$ has a $\mathrm{PBW}$ basis consisting of

$$
\left(\mathrm{x}_{1} \otimes 1\right)^{a_{1}} \cdots\left(\mathrm{x}_{n} \otimes 1\right)^{a_{n}}\left(1 \otimes \mathrm{x}_{1}\right)^{b_{1}} \cdots\left(1 \otimes \mathrm{x}_{n}\right)^{b_{n}}
$$

for $a_{i}, b_{i} \geq 0$, therefore the hypothesis of Corollary 3.8 holds. 


\subsection{Example: the quantum plane.}

Definition 3.10. The coordinate ring of the quantum plane is the $k(q)$-algebra $\mathrm{A}$ generated by $\mathrm{x}, \mathrm{y}$ subject to the relation $\mathrm{xy}=q \mathrm{yx}$.

A is graded with $|x|=|y|=1$; it is a quadratic algebra with basis consisting of all elements $\mathrm{x}^{a} \mathrm{y}^{b}$ with $a, b \geq 0$. The $k\left[q^{ \pm 1}\right]$-form $\mathcal{A}$ generated by $\mathrm{x}, \mathrm{y}$ has semiclassical limit $A$ which is polynomial on the images $x, y$ of $\mathrm{x}, \mathrm{y}$ and has Poisson structure determined by $\{x, y\}=x y$. By Lemma 2.2 the Poisson enveloping algebra $P(A)$ is generated by $x, y$ and $\Omega(x), \Omega(y)$ subject to

$$
\begin{gathered}
x y=y x, \Omega(x) y-y \Omega(x)=x y, \Omega(y) x-x \Omega(y)=-x y \\
\Omega(x) \Omega(y)-\Omega(y) \Omega(x)=x \Omega(y)+y \Omega(x)
\end{gathered}
$$

Theorem 3.9 shows $\mathrm{A}^{e}$ is a $q$-deformation of $P(A)$ via the $k\left[q^{ \pm 1}\right]$-form generated by $\mathrm{x}$, y and $\tilde{\Omega}(\mathrm{x}), \tilde{\Omega}(\mathrm{y})$. By Lemma 3.4, the following relations hold in $A^{e}$ :

$$
\begin{aligned}
\mathrm{xy}=q \mathrm{yx}, \tilde{\Omega}(\mathrm{x}) \mathrm{y}-\mathrm{y} \tilde{\Omega}(\mathrm{x}) & =\mathrm{xy}, \tilde{\Omega}(\mathrm{y}) \mathrm{x}-\mathrm{x} \tilde{\Omega}(\mathrm{y})=-\mathrm{xy} \\
q \tilde{\Omega}(\mathrm{x}) \tilde{\Omega}(\mathrm{y})-\tilde{\Omega}(\mathrm{x}) \tilde{\Omega}(\mathrm{y}) & =\mathrm{x} \tilde{\Omega}(\mathrm{y})+\mathrm{y} \tilde{\Omega}(\mathrm{x})
\end{aligned}
$$

In fact by counting the dimension of the relation space they are sufficient to give a presentation of $A^{e}$.

\subsection{Example: $2 \times 2$ quantum matrices.}

Definition 3.11. The algebra of $2 \times 2$ quantum matrices $\mathrm{M}$ is the $k(q)$-algebra generated by a, b, c, d subject to the relations

$$
\begin{gathered}
\mathrm{ab}=q \mathrm{ba} \quad \mathrm{ac}=q \mathrm{ca} \quad \mathrm{bc}=\mathrm{cb} \quad \mathrm{bd}=q \mathrm{db} \quad \mathrm{cd}=q \mathrm{dc} \\
\mathrm{ad}-\mathrm{da}=\left(q-q^{-1}\right) \mathrm{bc} .
\end{gathered}
$$

$M$ is graded with a, b, c, $d$ in degree 1 ; it is a quadratic algebra which admits a basis consisting of all elements $\mathrm{a}^{i} \mathrm{~b}^{j} \mathrm{c}^{k} \mathrm{~d}^{l}$ for $i, j, k, l \geq 0$.

The $k\left[q^{ \pm 1}\right]$-subalgebra $\mathcal{M}$ generated by a, b, c, d has semiclassical limit $M$ which is polynomial on the images $a, b, c, d$ of a, b, c, d with Poisson structure determined by

$$
\begin{array}{lll}
\{a, b\}=a b & \{a, c\}=a c & \{a, d\}=2 b c \\
\{b, c\}=0 & \{b, d\}=b d & \{c, d\}=c d .
\end{array}
$$


By Lemma 2.2 the Poisson enveloping algebra $P(M)$ is generated by $a, b, c, d$, $\Omega(a), \Omega(b), \Omega(c), \Omega(d)$ subject to relations saying that $a, b, c, d$ commute, and

$$
\begin{array}{ll}
\Omega(a) a-a \Omega(a)=0 & \Omega(a) b-b \Omega(a)=a b \\
\Omega(a) c-c \Omega(a)=a c & \Omega(a) d-d \Omega(a)=2 b c \\
\Omega(b) a-a \Omega(b)=-a b & \Omega(b) b-b \Omega(b)=0 \\
\Omega(b) c-c \Omega(b)=0 & \Omega(b) d-d \Omega(b)=b d \\
\Omega(c) a-a \Omega(c)=-a c & \Omega(c) b-b \Omega(c)=0 \\
\Omega(c) c-c \Omega(c)=0 & \Omega(c) d-d \Omega(c)=-c d \\
\Omega(d) a-a \Omega(d)=-2 b c & \Omega(d) b-b \Omega(d)=-b d \\
\Omega(d) c-c \Omega(d)=-c d & \Omega(d) d-d \Omega(d)=0 \\
\Omega(a) \Omega(b)-\Omega(b) \Omega(a)=a \Omega(b)+b \Omega(a) \\
\Omega(a) \Omega(c)-\Omega(c) \Omega(a)=a \Omega(c)+c \Omega(a) \\
\Omega(a) \Omega(d)-\Omega(d) \Omega(a)=2 b \Omega(c)+2 c \Omega(b) \\
\Omega(b) \Omega(c)-\Omega(c) \Omega(b)=0 \\
\Omega(b) \Omega(d)-\Omega(d) \Omega(b)=b \Omega(d)+d \Omega(b) \\
\Omega(c) \Omega(d)-\Omega(d) \Omega(c)=c \Omega(d)+d \Omega(c)
\end{array}
$$

Theorem 3.9 shows $\mathrm{M}^{e}$ is a $q$-deformation of $P(M)$ via the $k\left[q^{ \pm 1}\right]$-form generated by a, b, c, d and $\tilde{\Omega}(\mathrm{a}), \tilde{\Omega}(\mathrm{b}), \tilde{\Omega}(\mathrm{c}), \tilde{\Omega}(\mathrm{d})$.

By Lemma 3.4, the following relations hold in $\mathrm{M}^{e}$ :

$$
\begin{array}{ll}
\tilde{\Omega}(\mathrm{a}) \mathrm{a}-\mathrm{a} \tilde{\Omega}(\mathrm{a})=0 & \tilde{\Omega}(\mathrm{a}) \mathrm{b}-\mathrm{b} \tilde{\Omega}(\mathrm{a})=\mathrm{ab} \\
\tilde{\Omega}(\mathrm{a}) \mathrm{c}-\mathrm{c} \tilde{\Omega}(\mathrm{a})=\mathrm{ac} & \tilde{\Omega}(\mathrm{a}) \mathrm{d}-\mathrm{d} \tilde{\Omega}(\mathrm{a})=\left(1+q^{-1}\right) \mathrm{bc} \\
\tilde{\Omega}(\mathrm{b}) \mathrm{a}-\mathrm{a} \tilde{\Omega}(\mathrm{b})=-\mathrm{ab} & \tilde{\Omega}(\mathrm{b}) \mathrm{b}-\mathrm{b} \tilde{\Omega}(\mathrm{b})=0 \\
\tilde{\Omega}(\mathrm{b}) \mathrm{c}-\mathrm{c} \tilde{\Omega}(\mathrm{b})=0 & \tilde{\Omega}(\mathrm{b}) \mathrm{d}-\mathrm{d} \tilde{\Omega}(\mathrm{b})=\mathrm{bd} \\
\tilde{\Omega}(\mathrm{c}) \mathrm{a}-\mathrm{a} \tilde{\Omega}(\mathrm{c})=-\mathrm{ac} & \tilde{\Omega}(\mathrm{c}) \mathrm{b}-\mathrm{b} \tilde{\Omega}(\mathrm{c})=0 \\
\tilde{\Omega}(\mathrm{c}) \mathrm{c}-\mathrm{c} \tilde{\Omega}(\mathrm{c})=0 & \tilde{\Omega}(\mathrm{c}) \mathrm{d}-\mathrm{d} \tilde{\Omega}(\mathrm{c})=-\mathrm{cd} \\
\tilde{\Omega}(\mathrm{d}) \mathrm{a}-\mathrm{a} \tilde{\Omega}(\mathrm{d})=-\left(1+q^{-1}\right) \mathrm{bc} & \tilde{\Omega}(\mathrm{d}) \mathrm{b}-\mathrm{b} \tilde{\Omega}(\mathrm{d})=-\mathrm{bd} \\
\tilde{\Omega}(\mathrm{d}) \mathrm{c}-\mathrm{c} \tilde{\Omega}(\mathrm{d})=-\mathrm{cd} & \tilde{\Omega}(\mathrm{d}) \mathrm{d}-\mathrm{d} \tilde{\Omega}(\mathrm{d})=0 \\
q \tilde{\Omega}(\mathrm{a}) \tilde{\Omega}(\mathrm{b})-\tilde{\Omega}(\mathrm{b}) \tilde{\Omega}(\mathrm{a})=\mathrm{a} \tilde{\Omega}(\mathrm{b})+\mathrm{b} \tilde{\Omega}(\mathrm{a}) \\
q \tilde{\Omega}(\mathrm{a}) \tilde{\Omega}(\mathrm{c})-\tilde{\Omega}(\mathrm{c}) \tilde{\Omega}(\mathrm{a})=\mathrm{a} \tilde{\Omega}(\mathrm{c})+\mathrm{c} \tilde{\Omega}(\mathrm{a}) \\
\tilde{\Omega}(\mathrm{a}) \tilde{\Omega}(\mathrm{d})-\tilde{\Omega}(\mathrm{d}) \tilde{\Omega}(\mathrm{a})=\left(1+q^{-1}\right) \mathrm{b} \tilde{\Omega}(\mathrm{c})+\left(1+q^{-1}\right) \mathrm{c} \tilde{\Omega}(\mathrm{b})-\left(q-q^{-1}\right) \tilde{\Omega}(\mathrm{b}) \tilde{\Omega}(\mathrm{c}) \\
\tilde{\Omega}(\mathrm{b}) \tilde{\Omega}(\mathrm{c})-\tilde{\Omega}(\mathrm{c}) \tilde{\Omega}(\mathrm{b})=0 & \\
q \tilde{\Omega}(\mathrm{b}) \tilde{\Omega}(\mathrm{d})-\tilde{\Omega}(\mathrm{d}) \tilde{\Omega}(\mathrm{b})=\mathrm{b} \tilde{\Omega}(\mathrm{d})+\mathrm{d} \tilde{\Omega}(\mathrm{b}) \\
q \tilde{\Omega}(\mathrm{c}) \tilde{\Omega}(\mathrm{d})-\tilde{\Omega}(\mathrm{d}) \tilde{\Omega}(\mathrm{c})=\mathrm{c} \tilde{\Omega}(\mathrm{d})+\mathrm{d} \tilde{\Omega}(\mathrm{c})
\end{array}
$$

\section{Koszul algebras and modules}

We refer to [13] for general background on quadratic and Koszul algebras. Recall that a graded $k$-algebra $\Lambda$ is called quadratic if it there is a finite-dimensional vector space $V$ and a subspace $R \leq V \otimes_{k} V$ such that $\Lambda \cong T(V) /(R)$ where $T(V)$ 
denotes the tensor algebra. The quadratic dual $\Lambda^{!}$of $\Lambda$ is $T\left(V^{*}\right) /\left(R^{\perp}\right)$ where $V^{*}=\operatorname{Hom}_{k}(V, k)$ and $R^{\perp}$ is the image of the annihilator of $R$ under the canonical isomorphism of $\left(V \otimes_{k} V\right)^{*}$ with $V^{*} \otimes_{k} V^{*}$.

Example 4.1. Let $M$ be the algebra of $2 \times 2$ quantum matrices defined in Section 3.2. The quadratic dual $M^{!}$of $M$ is generated by $a^{*}, b^{*}, c^{*}, d^{*}$ subject to

$$
\begin{gathered}
\mathrm{a}^{* 2}, \mathrm{~b}^{* 2}, \mathrm{c}^{* 2}, \mathrm{~d}^{* 2}, \mathrm{~b}^{*} \mathrm{c}^{*}+\mathrm{c}^{*} \mathrm{~b}^{*}+\left(q-q^{-1}\right) \mathrm{a}^{*} \mathrm{~d}^{*} \\
q \mathrm{a}^{*} \mathrm{~b}^{*}+\mathrm{b}^{*} \mathrm{a}^{*}, q \mathrm{a}^{*} \mathrm{c}^{*}+\mathrm{c}^{*} \mathrm{a}^{*}, \mathrm{a}^{*} \mathrm{~d}^{*}+\mathrm{d}^{*} \mathrm{a}^{*}, q \mathrm{~b}^{*} \mathrm{~d}^{*}+\mathrm{d}^{*} \mathrm{~b}^{*}, q \mathrm{c}^{*} \mathrm{~d}^{*}+\mathrm{d}^{*} \mathrm{c}^{*} .
\end{gathered}
$$

A graded left $\Lambda$-module $M$ is called quadratic if it is isomorphic as a graded module to one of the form $\left(\Lambda \otimes_{k} M_{0}\right) / \Lambda H$ where $M_{0}$ is a finite-dimensional vector space, $H \leq \Lambda_{1} \otimes M_{0}$, and $M_{0}$ is homogeneous with respect to the grading. The quadratic dual $M^{!}$of $M$ is the left $\Lambda^{!}$-module $\left(\Lambda^{!} \otimes_{k} M_{0}^{*}\right) /\left(\Lambda^{!} H^{\perp}\right)$ where $H^{\perp}$ denotes the image of the annihilator of $H$ under the canonical isomorphism between $\left(\Lambda_{1} \otimes_{k} M_{0}\right)$ and $\Lambda_{1}^{*} \otimes_{k} M_{0}^{*}=\left(\Lambda^{!}\right)_{1} \otimes_{k} M_{0}^{*}$. If $M$ and $N$ are graded left $\Lambda$-modules, $\operatorname{Ext}_{\Lambda}^{*}(M, N)$ is bigraded, by homological degree and by internal degree. We write $\operatorname{Ext}_{\Lambda}^{i j}(M, N)$ for the part with homological degree $i$ and internal degree $j$. Write $k$ for the trivial $\Lambda$-module $\Lambda / \bigoplus_{i>0} \Lambda_{i}$. A quadratic algebra $\Lambda$ is called Koszul if $\operatorname{Ext}_{\Lambda}^{i j}(k, k)$ is zero whenever $i \neq j$, in which case $\operatorname{Ext}_{\Lambda}^{*}(k, k) \cong \Lambda^{!}$as algebras. A graded module $M$ over a Koszul algebra $\Lambda$ is called Koszul if $\operatorname{Ext}_{\Lambda}^{i j}(M, k)$ is zero if $i \neq j$, or equivalently if $M$ admits a linear projective resolution, that is, a projective resolution $P_{*} \rightarrow M$ such that $P_{i}$ is generated by its component of degree $i$.

4.1. Hochschild cohomology of Koszul algebras. If $\Lambda$ is Koszul then $\Lambda^{e}$ is Koszul: $\Lambda^{\text {op }}$ is Koszul by [13, remark on p.20], and tensor products of Koszul algebras are Koszul by [13, Corollary 3.1.2]. Furthermore $\Lambda$ is a Koszul $\Lambda^{e}$-module by [6, Corollary 2.2].

Lemma 4.2. The quadratic dual $\left(\Lambda^{e} \Lambda\right)^{!}$of the $\Lambda^{e}$-module $\Lambda$ is isomorphic as a vector space to the dual quadratic algebra $\Lambda$ !.

Proof. Let $\Lambda=T(V) /(R)$ so that $\Lambda^{e}=T\left(V \oplus V^{\prime}\right) /\left(R \oplus R^{\prime} \oplus C\right)$ where $V^{\prime}$ is isomorphic to $V$ via a map that sends $v \in V$ to $v^{\prime}, R^{\prime}$ is the image of $R$ under the twist map $\tau: v \otimes w \mapsto w^{\prime} \otimes v^{\prime}$ and $C$ has basis $x_{i} \otimes x_{j}^{\prime}-x_{j}^{\prime} \otimes x_{i}$ where $x_{1}, \ldots, x_{n}$ is some fixed basis of $V$.

Thus $\left(\Lambda^{e}\right)$ ! is isomorphic to $T\left(V^{*} \oplus V^{\prime *}\right) /\left(R^{\perp} \oplus R^{\prime \perp} \oplus D\right)$ where $D$ has a basis consisting of all tensors of the form $x_{i}^{*} \otimes x_{j}^{\prime *}+x_{j}^{\prime *} \otimes x_{i}^{*}$ where the $x_{i}^{*}$ are the basis of $V^{*}$ dual to $x_{1}, \ldots, x_{n}$. It follows $\left(\Lambda^{e}\right)^{!} \cong \Lambda^{!} \hat{\otimes}_{k}\left(\Lambda^{!}\right)^{\text {op }}$ where $\hat{\otimes}_{k}$ denotes the graded commutative tensor product: $\left(\lambda_{1} \hat{\otimes} \mu_{1}\right) \cdot\left(\lambda_{2} \hat{\otimes} \mu_{2}\right)=(-1)^{m n} \lambda_{1} \lambda_{2} \hat{\otimes} \mu_{1} \mu_{2}$ for $\lambda_{2}, \mu_{1}$ homogeneous of degrees $m$ and $n$ respectively.

As a $\Lambda^{e}=T\left(V \oplus V^{\prime}\right) /\left(R \oplus R^{\prime} \oplus C\right)$-module, $\Lambda=\Lambda^{e} /\left(x_{i}-x_{i}^{\prime}: i=1, \ldots, n\right)$. Therefore the quadratic dual of $\Lambda^{e} \Lambda$ is $\left(\Lambda^{e}\right)^{!} /\left(x_{i}^{*}+x_{i}^{\prime *}: i=1, \ldots, n\right)$. This 
ideal corresponds to the ideal $\left(x_{i}^{*} \otimes 1+1 \otimes x_{i}^{* *}\right)$ under the isomorphism between $T\left(V \oplus V^{\prime}\right) /\left(R \oplus R^{\prime} \oplus C\right)$ and $\Lambda^{!} \hat{\otimes}_{k}\left(\Lambda^{!}\right)^{\text {op }}$. There is an exact sequence of $\Lambda ! \hat{\otimes}\left(\Lambda^{!}\right)^{\mathrm{op}}$-modules

$$
0 \rightarrow\left(x_{i}^{*} \otimes 1+1 \otimes x_{i}^{\prime *}\right) \rightarrow \Lambda^{!} \hat{\otimes}_{k}\left(\Lambda^{!}\right)^{\mathrm{op}} \rightarrow \hat{\Lambda}^{!} \rightarrow 0
$$

where $\hat{\Lambda}^{!}$is the $\Lambda^{!} \hat{\otimes}_{k}\left(\Lambda^{!}\right)^{\text {op }}$-module which is $\Lambda^{!}$as a vector space, and with action $\left(\lambda \hat{\otimes} \mu^{\prime}\right) \cdot x=(-1)^{|\mu||x|+|\mu|(|\mu|+1) / 2} \lambda x \mu$ for $\lambda, x, \mu \in \Lambda^{!}$, homogeneous, and the map $\Lambda^{!} \hat{\otimes}_{k}\left(\Lambda^{!}\right)^{\text {op }} \rightarrow \hat{\Lambda}^{!}$is determined by $1 \otimes 1 \mapsto 1$. This completes the proof.

Definition 4.3. Let $M$ be a Koszul left-module for the Koszul $k$-algebra $\Gamma=$ $T(V) /(R)$ and let $e_{\Gamma} \in \Gamma \otimes_{k} \Gamma^{!}$be $\sum_{i} v_{i} \otimes v_{i}^{*}$ where $v_{i}$ runs over a basis of $V$ and $v_{i}^{*}$ is the corresponding dual basis element of $V^{*}=\Gamma_{1}^{!}$. Then the Koszul resolution $K_{\Gamma}(M)$ is $\Gamma \otimes_{k}\left(M^{!}\right)^{*}$ with differential given by right-multiplication by $e_{\Gamma}$, and the Koszul cocomplex $\bar{K}_{\Gamma}(M)$ is $M \otimes_{k} M^{!}$with differential given by left-multiplication by $e_{\Gamma}$.

[13, §2.3] shows that $K_{\Gamma}(M)$ is a minimal free resolution of $M$, so that $\operatorname{Ext}_{\Gamma}^{*}(M, M)$ is computed by cohomology of the cocomplexes

$$
\operatorname{Hom}_{\Gamma}\left(\Gamma \otimes_{k}\left(M^{!}\right)^{*}, M\right) \cong \operatorname{Hom}_{k}\left(\left(M^{!}\right)^{*}, M\right) \cong M \otimes_{k} M^{!}=\bar{K}_{\Gamma}(M) .
$$

Consider the special case when $\Gamma=\Lambda^{e}$ for some Koszul algebra $\Lambda$ and $M={ }_{\Lambda^{e}} \Lambda$. By Lemma 4.2, $\left(\Lambda^{e} \Lambda\right)^{!}$is $\hat{\Lambda}^{!}$so $\bar{K}_{\Lambda^{e}}(\Lambda)$ is isomorphic to $\Lambda \otimes_{k} \Lambda^{\text {! }}$ as a vector space. The corresponding differential is

$$
\lambda \otimes \mu \mapsto \sum_{i}\left(x_{i} \lambda \otimes x_{i}^{*} \mu+(-1)^{|\mu|+1} \lambda x_{i} \otimes \mu x_{i}^{*}\right)
$$

for $\mu$ homogeneous of degree $|\mu|$ - see [16, p.5]. This differential makes $\Lambda \otimes_{k} \Lambda^{!}$, with its natural multiplication, into a differential graded algebra.

This means that the Hochschild cohomology ring $\operatorname{HH}(\Lambda)=\operatorname{Ext}_{\Lambda^{e}}^{*}(\Lambda, \Lambda)$ has two multiplications: one it inherits as the cohomology of the differential graded algebra $\Lambda \otimes_{k} \Lambda^{!}$and one from the Yoneda product on Ext. We want to show that they agree.

Lemma 4.4. The cohomology of the differential graded algebra $\Lambda \otimes_{k} \Lambda$ ! equipped with the differential (4.1) is isomorphic as an algebra to $\mathrm{HH}^{*}(\Lambda)$.

Proof. We already know that the cohomology of $\Lambda \otimes_{k} \Lambda$ ! with this differential agrees with $\mathrm{HH}^{*}(\Lambda)$ as a vector space. To show that the two products are the same we need to examine the Koszul resolution more closely. If $\Lambda=T(V) /(R)$ then $\Lambda_{m}^{!}$is equal to

$$
\frac{\left(V^{*}\right)^{\otimes m}}{\sum_{i=0}^{m-2}\left(V^{*}\right)^{\otimes i} \otimes R^{\perp} \otimes\left(V^{*}\right)^{\otimes(m-2-i)}}
$$


(the denominator is to be interpreted as zero if $m=0$ or 1). For $m \geq 2$ the denominator is the annihilator of

$$
N_{m}=\bigcap_{i=0}^{m-2} V^{\otimes i} \otimes R \otimes V^{\otimes(m-2-i)}
$$

so we can identify $\left(\Lambda_{m}^{!}\right)^{*}$ with $N_{m}$, and the Koszul resolution $K_{\Lambda} e(\Lambda)$ of $\Lambda$ over $\Lambda^{e}$ can be written as

$$
K_{\Lambda^{e}}(\Lambda)_{m}=\Lambda \otimes N_{m} \otimes \Lambda
$$

where $N_{1}=V$ and $N_{0}=k$. As before let $x_{1}, \ldots, x_{n}$ be a basis of $V$, and given a sequence $\mathbf{i}=\left(i_{1}, \ldots, i_{m}\right)$ write $x_{\mathbf{i}}$ for $x_{i_{1}} \otimes \cdots \otimes x_{i_{m}} \in V^{\otimes m}$. The differential on (4.2) is

$$
1 \otimes\left(\sum_{\mathbf{i}} \alpha_{\mathbf{i}} x_{\mathbf{i}}\right) \otimes 1 \mapsto \sum_{\mathbf{i}} \alpha_{\mathbf{i}}\left(x_{i_{1}} \otimes x_{\left(i_{2}, \ldots, i_{m}\right)} \otimes 1-1 \otimes x_{\left(i_{1}, \ldots, i_{m}-1\right.} \otimes x_{i_{m}}\right)
$$

where $\alpha_{\mathbf{i}} \in k$.

Write $\mathbb{B}_{*}$ for the standard (bar) complex of $\Lambda$ [3, IX.6], whose $m$ th term is $\mathbb{B}_{m}=$ $\Lambda^{\otimes(m+2)}$. The inclusion $V \hookrightarrow \Lambda$ induces a map

$$
\iota: K_{\Lambda^{e}}(\Lambda)_{*} \rightarrow \mathbb{B}_{*}
$$

which is a morphism of chain complexes [16, Proposition 3.3]. The bar complex of $\Lambda$ admits a comultiplication $\Delta: \mathbb{B}_{*} \rightarrow \mathbb{B}_{*} \otimes_{\Lambda} \mathbb{B}_{*}$ defined by

$$
\Delta\left(\lambda \otimes y_{\mathbf{i}} \otimes \mu\right)=\sum_{r}\left(\lambda \otimes y_{\left(i_{1}, \ldots, i_{r}\right)} \otimes 1\right) \otimes\left(1 \otimes y_{\left(i_{m+1}, \ldots, i_{m}\right)} \otimes \mu\right)
$$

where $y_{\mathbf{i}}=y_{i_{1}} \otimes \cdots \otimes y_{i_{m}} \in \Lambda^{\otimes m}$. We will show that

$$
\Delta(\operatorname{im} \iota) \subseteq(\operatorname{im} \iota) \otimes_{\Lambda}(\operatorname{im} \iota)
$$

so that $\Delta$ induces a comultiplication on $K_{\Lambda^{e}}(\Lambda)$. This is equivalent to proving that if $\sum_{\mathbf{i}} \alpha_{\mathbf{i}} x_{\mathbf{i}} \in N_{m}$ then

$$
\sum_{\mathbf{i}} \alpha_{\mathbf{i}} x_{\left(i_{1}, \ldots, i_{r}\right)} \otimes x_{\left(i_{r+1}, \ldots, i_{m}\right)} \in N_{r} \otimes N_{m-r}
$$

for any $r \leq m$. Corollary 3.3 of [14] says that for any sequence $\mathbf{j}$ of length $m-r$,

$$
\sum_{\mathrm{i}:\left(i_{r+1}, \ldots, i_{m}\right)=\mathbf{r}} \alpha_{\mathbf{i}} x_{\left(i_{1}, \ldots, i_{r}\right)} \in N_{r}
$$

Thus

$\sum_{\mathbf{i}} \alpha_{\mathbf{i}} x_{\left(i_{1}, \ldots, i_{r}\right)} \otimes x_{\left(i_{r+1}, \ldots, i_{m}\right)}=\sum_{\mathbf{j}}\left(\sum_{\mathbf{i}:\left(i_{r+1}, \ldots, i_{m}\right)=\mathbf{r}} \alpha_{\mathbf{i}} x_{\left(i_{1}, \ldots, i_{r}\right)}\right) \otimes x_{\mathbf{j}} \in N_{r} \otimes V^{\otimes m-r}$. 
Similarly it lies in $V^{\otimes r} \otimes N_{m-r}$, so in

$$
\left(N_{r} \otimes V^{\otimes m-r}\right) \cap\left(V^{\otimes r} \otimes N_{m-r}\right)=N_{r} \otimes N_{m-r}
$$

completing the proof of (4.3).

If we identify $\Lambda \otimes_{\Lambda} \Lambda$ with $\Lambda$ then $\mathbb{B}_{*} \otimes_{\Lambda} \mathbb{B}_{*}$ is a free resolution of $\Lambda$ and $\Delta$ is a chain map lifting the identity map on $\Lambda$. Because of (4.3) the same holds for $K_{\Lambda^{e}}(\Lambda)$. [2, p.4] point out that the Yoneda product on $\mathrm{HH}(\Lambda)$ can be computed as follows: if $f: K_{\Lambda^{e}}(\Lambda)_{r} \rightarrow \Lambda$ and $g: K_{\Lambda^{e}}(\Lambda)_{s} \rightarrow \Lambda$ are cocycles, the product of the cohomology elements they represent is represented by $f * g=\left(f \otimes_{\Lambda} g\right) \circ \Delta$ where $f \otimes_{\Lambda} g: K_{\Lambda^{e}}(\Lambda)_{r} \otimes_{\Lambda} K_{\Lambda^{e}}(\Lambda)_{s} \rightarrow \Lambda$ is $\kappa \otimes \kappa^{\prime} \mapsto f(\kappa) g\left(\kappa^{\prime}\right)$.

There is a linear isomorphism $\phi: \Lambda \otimes \Lambda^{!} \mapsto \operatorname{Hom}_{\Lambda^{e}}\left(K_{\Lambda^{e}}(\Lambda)_{*}, \Lambda\right)$ that sends $\lambda \otimes \mu$ to

$$
1 \otimes \mathbf{n} \otimes 1 \mapsto\langle\mu, \mathbf{n}\rangle \lambda
$$

where $\mu \in \Lambda_{m}^{!}, \mathbf{n} \in N_{m}$ and $\langle-,-\rangle$ is the pairing between $\Lambda_{m}^{!}$and $N_{m}$. We will show this is is a homomorphism of algebras when $\operatorname{Hom}_{\Lambda^{e}}\left(K_{\Lambda^{e}}(\Lambda)_{*}, \Lambda\right)$ is equipped with the product $*$.

Since the product in $\Lambda^{!}$is induced by tensor multiplication in $T\left(V^{*}\right)$, if $\mu=$ $\hat{\mu}+\operatorname{Ann}\left(N_{r}\right) \in \Lambda_{r}^{!}$and $\mu^{\prime}=\hat{\mu}^{\prime}+\operatorname{Ann}\left(N_{s}\right) \in \Lambda_{s}^{!}$then

$$
\phi\left(\lambda \lambda^{\prime} \otimes \mu \mu^{\prime}\right)\left(1 \otimes\left(\sum_{\mathbf{i}} \alpha_{\mathbf{i}} x_{\mathbf{i}}\right) \otimes 1\right)=\sum_{\mathbf{i}} \alpha_{\mathbf{i}} \hat{\mu}\left(x_{\left(i_{1}, \ldots, i_{r}\right)}\right) \hat{\mu}^{\prime}\left(x_{\left(i_{r+1}, \ldots, i_{r+s}\right)}\right) \lambda \lambda^{\prime} .
$$

On the other hand

$$
\begin{aligned}
& \left(\phi(\lambda \otimes \mu) * \phi\left(\lambda^{\prime} \otimes \mu^{\prime}\right)\right)\left(1 \otimes\left(\sum_{\mathbf{i}} \alpha_{\mathbf{i}} x_{\mathbf{i}}\right) \otimes 1\right) \\
& =\phi(\lambda \otimes \mu) \otimes \phi\left(\lambda^{\prime} \otimes \mu^{\prime}\right) \sum_{\mathbf{i}} \alpha_{\mathbf{i}}\left(1 \otimes x_{\left(i_{1}, \ldots, i_{r}\right)} \otimes 1\right) \otimes\left(1 \otimes x_{\left(i_{r+1}, \ldots, i_{r+s}\right)} \otimes 1\right) \\
& \quad=\sum_{\mathbf{i}} \alpha_{\mathbf{i}} \hat{\mu}\left(x_{\left(i_{1}, \ldots, i_{r}\right)}\right) \hat{\mu}^{\prime}\left(x_{\left(i_{r+1}, \ldots, i_{r+s}\right)}\right) \lambda \lambda^{\prime}
\end{aligned}
$$

This completes the proof.

4.2. Quadratic Poisson enveloping algebras are Koszul. A polynomial Poisson algebra $A=k\left[x_{1}, \ldots, x_{n}\right]$ is a left Poisson module over itself in the obvious way. $A$ is therefore a $P(A)$-module, isomorphic to the quotient of $P(A)$ by the left ideal generated by the $\Omega\left(y_{i}\right)$.

Lemma 4.5. Let $A=k\left[x_{1}, \ldots, x_{n}\right]$ be a polynomial algebra, graded with each $x_{i}$ in degree one, equipped with a Poisson bracket $\{-,-\}$ such that $\left\{x_{i}, x_{j}\right\} \in A_{2}$ for all $i$ and $j$. Then $P(A)$ is a Koszul algebra and $A$ is a Koszul $P(A)$-module.

A Poisson algebra or bracket with this property will be called quadratic. 
Proof. If we place all $y_{i}$ and $\Omega\left(y_{i}\right)$ in degree one, the defining relations (2.2), (2.3) and (2.4) of $P(A)$ are homogeneous of degree two. Applying Corollary 2.3 shows that $P(A)$ is a quadratic algebra with a PBW basis, and such algebras are Koszul by a result of Priddy [13, Theorem 4.3.1].

When $\Omega(A)$ is a projective $A$-module, which holds when $A$ is polynomial, Huebschmann [8, p.66] points out that $P(A) \otimes_{A} \Lambda_{A}^{*}(\Omega(A))$ with the CartanChevalley-Eilenberg differential is a projective resolution of $A$ over $P(A)$. Since this is clearly a linear resolution, $A$ is a Koszul $P(A)$-module by [13, p.20].

Corollary 4.6. $P(A)^{!}$is generated by $y_{i}^{*}$ and $\Omega\left(y_{i}^{*}\right)$ subject to

$$
\begin{gathered}
\Omega\left(y_{i}\right)^{*} \Omega\left(y_{j}\right)^{*}+\Omega\left(y_{j}\right)^{*} \Omega\left(y_{i}\right)^{*} \quad 1 \leq i \leq j \leq n \\
\Omega\left(y_{m}\right)^{*} y_{n}^{*}+y_{n}^{*} \Omega\left(y_{m}\right)^{*}+\sum_{i<j} \frac{\partial\left\{x_{i}, x_{j}\right\}}{\partial x_{m} \partial x_{n}} \Omega\left(y_{i}\right)^{*} \Omega\left(y_{j}\right)^{*} \\
y_{m}^{*} y_{n}^{*}+y_{n}^{*} y_{m}^{*}-\sum_{i<j} \frac{\partial\left\{x_{i}, x_{j}\right\}}{\partial x_{m} \partial x_{n}}\left(\Omega\left(y_{i}\right)^{*} y_{j}^{*}-\Omega\left(y_{j}\right)^{*} y_{i}^{*}\right) .
\end{gathered}
$$

The subalgebra $O=\left\langle\Omega\left(y_{1}\right)^{*}, \ldots, \Omega\left(y_{n}\right)^{*}\right\rangle$ of $P(A)^{!}$is exterior of rank $n$.

Proof. The given presentation for $P(A)^{!}$follows from the presentation of $P(A)$ given by relations (2.2), (2.3) and (2.4).

[13, Corollary 2.2] says that if $M$ is a Koszul module over a Koszul algebra $\Lambda$ and $M^{!}, \Lambda^{!}$are their Koszul duals then $h_{\Lambda}(t) h_{\Lambda^{!}}(-t)=1$ and $h_{M^{!}}(t)=h_{\Lambda^{!}}(t) h_{M}(-t)$ where $h_{M}(t), h_{\Lambda}(t), h_{M !}(t), h_{\Lambda}$ ! are the Hilbert series of $M, \Lambda, M^{!}, \Lambda^{!}$respectively. Applying this with $M=A$ whose Hilbert series is $(1-t)^{-n}$ and $\Lambda=P(A)$ whose Hilbert series is $(1-t)^{-2 n}$ shows that $h_{P(A)} !=(1+t)^{2 n}$ and the Koszul dual of the $P(A)$-module $A$ has Hilbert series $(1+t)^{n}$.

By definition, $A^{!}=P(A)^{!} / P(A)^{!}\left(y_{1}^{*}, \ldots, y_{n}^{*}\right)$ and repeatedly using the second relation shows this quotient is spanned by $O+P(A)^{!}\left(y_{1}^{*}, \ldots, y_{n}^{*}\right)$. But $\operatorname{dim} A^{!}=2^{n}$, so $\operatorname{dim} O \geq 2^{n}$. Since $O$ is certainly a quotient of an exterior algebra of rank $n$ we in fact have $\operatorname{dim} O=2^{n}$. The last statement follows.

At the moment $A^{!}$could mean two different things: the Koszul dual of the algebra $A$ and the quadratic dual of the $P(A)$-module $A$. In what follows $A^{\text {! }}$ always refers to the algebra $O$ with $P(A)$-module structure induced by the vector space isomorphism between $O$ and $P(A)^{!} / P(A)^{!}\left(y_{1}^{*}, \ldots, y_{n}^{*}\right)$ from the proof of the previous corollary.

Remark 4.7. The quadratic dual of $P(A)$ can be described as follows. A Poisson superalgebra (or graded Poisson algebra) $B$ is a graded-commutative algebra, that is $a b=(-1)^{|a||b|} b a$ for homogeneous elements $a, b$ of $B$, equipped with a bilinear 
bracket $\{-,-\}$ such that

$$
\begin{gathered}
\{a, b\}=(-1)^{1+|a||b|}\{b, a\} \\
(-1)^{|a||c|}\{a,\{b, c\}\}+(-1)^{|a||b|}\{b,\{c, a\}\}+(-1)^{|b||c|}\{c,\{a, b\}\}=0 \\
\{a, b c\}=\{a, b\} c+(-1)^{|a||b|} b\{a, c\}
\end{gathered}
$$

for all homogeneous $a, b, c \in B$.

A quadratic Poisson bracket on the polynomial algebra $A$ is determined by a map $b: \Lambda^{2}\left(A_{1}\right) \rightarrow S^{2}\left(A_{1}\right)$, where $\Lambda^{2}$ and $S^{2}$ are the exterior and symmetric squares and $A_{1}$ is the vector space spanned by the $x_{i}$. The dual map $b^{*}: S^{2}\left(A_{1}^{*}\right) \rightarrow \Lambda^{2}\left(A_{1}^{*}\right)$ allows us to define a quadratic Poisson superalgebra structure on the exterior algebra generated by the $x_{i}^{*}$ by

$$
\left\{x_{i}^{*}, x_{j}^{*}\right\}:=b^{*}\left(x_{i}^{*}, x_{j}^{*}\right) .
$$

The quadratic dual of $P(A)$ is isomorphic to the Poisson enveloping algebra of the exterior algebra generated by the $x_{i}^{*}$ with graded Poisson bracket determined by $b^{*}$.

Corollary 4.8. Let $A$ be a polynomial algebra with a quadratic Poisson bracket. Then the Koszul cocomplex $\bar{K}_{P(A)}(A)=A \otimes_{k} A^{!}$with its natural multiplication and differential $e_{P(A)}$ is a differential graded algebra whose cohomology is isomorphic as an algebra to $\operatorname{HP}^{*}(A)$.

Proof. We will show that $\bar{K}_{P(A)}(A)$ with differential $e_{P(A)}$ is isomorphic as a differential graded algebra to $\operatorname{Alt}_{A}^{*}(\Omega(A), A)$. As in Subsection 2.2 we identify this with $A \otimes \Lambda^{*}\left(V^{*}\right)$ where $V^{*}$ is the span of the $\Omega\left(x_{i}\right)^{*}$, and map $A \otimes_{k} \Lambda^{*}\left(V^{*}\right) \rightarrow$ $\bar{K}_{P(A)}(A)$ by $\phi: a \otimes \Omega\left(x_{i}\right)^{*} \mapsto a \otimes \Omega\left(y_{i}\right)^{*}$. This is clearly an isomorphism of algebras; we only have to show that it respects the differential. Since $A \otimes_{k} \Lambda^{*}\left(V^{*}\right)$ is generated as an algebra by the $x_{i} \otimes 1$ and $1 \otimes \Omega\left(x_{i}\right)^{*}$ it is enough to check that $\phi\left(\delta\left(x_{i} \otimes 1\right)\right)=e_{P(A)}\left(x_{1} \otimes 1\right)$ and $\phi\left(\delta\left(1 \otimes \Omega\left(x_{i}\right)^{*}\right)\right)=e_{P(A)}\left(1 \otimes \Omega\left(y_{i}\right)^{*}\right)$, where $\delta$ is the differential corresponding to the Cartan-Chevalley-Eilenberg differential $d$ on $\operatorname{Alt}_{A}^{*}(\Omega(A), A)$. The element $x_{i} \otimes 1 \in A \otimes_{k} \Lambda^{*}\left(V^{*}\right)$ corresponds to $f_{i}: 1 \otimes 1 \mapsto$ $x_{i} \in \operatorname{Alt}_{A}^{*}(\Omega(A), A)$, and

$$
d\left(f_{i}\right)\left(1 \otimes \Omega\left(x_{j}\right)\right)=\Omega\left(x_{j}\right) f(1 \otimes 1)=\left\{x_{j}, x_{i}\right\}
$$

so that $\delta\left(x_{i} \otimes 1\right)=\sum_{j}\left\{x_{j}, x_{i}\right\} \otimes \Omega\left(x_{j}\right)^{*}$, and the image of this under $\phi$ is $\sum_{j}\left\{x_{j}, x_{i}\right\} \otimes \Omega\left(y_{j}\right)^{*}$. Applying

$$
e_{P(A)}=\sum_{j}\left(y_{j} \otimes y_{j}^{*}+\Omega\left(y_{j}\right) \otimes \Omega\left(y_{j}^{*}\right)\right)
$$

to $x_{i} \otimes 1$ gives $\sum_{j} \Omega\left(y_{j}\right) \cdot x_{i} \otimes \Omega\left(y_{j}\right)^{*}=\sum_{j}\left\{x_{j}, x_{i}\right\} \otimes \Omega\left(y_{j}\right)^{*}$, so $\phi\left(\delta\left(x_{i} \otimes 1\right)\right)=$ $e_{P(A)}\left(x_{1} \otimes 1\right)$ holds. 
$1 \otimes \Omega\left(x_{i}\right)^{*}$ corresponds to the element $f_{i}$ of $\operatorname{Alt}_{A}(\Omega(A), A)$ that sends $1 \otimes \Omega\left(x_{j}\right)$ to 1 if $i=j$ and 0 otherwise. Therefore

$$
d f_{i}\left(1 \otimes \Omega\left(x_{j}\right) \wedge \Omega\left(x_{k}\right)\right)=-d f_{i}\left(1 \otimes_{A} \Omega\left(\left\{x_{j}, x_{k}\right\}\right)\right.
$$

which is equal to minus the coefficient of $\Omega\left(x_{i}\right)$ in $\Omega\left(\left\{x_{j}, x_{k}\right\}\right)$. Therefore

$$
\delta\left(1 \otimes \Omega\left(x_{i}\right)^{*}\right)=-\sum_{j<k} c_{i j k} \otimes \Omega\left(x_{j}\right)^{*} \wedge \Omega\left(x_{k}\right)^{*}
$$

where $c_{i j k}$ is the coefficient of $\Omega\left(x_{i}\right)$ in $\Omega\left(\left\{x_{j}, x_{k}\right\}\right)$. On the other hand, applying $e_{P(A)}$ to $1 \otimes \Omega\left(y_{i}\right)^{*}$ gives

$$
\sum_{r} y_{r} \otimes y_{r}^{*} \Omega\left(y_{i}\right)^{*}=-\sum_{j<k}\left(\sum_{r} y_{r} \frac{\partial\left\{x_{j}, x_{k}\right\}}{\partial x_{r} \partial x_{i}}\right) \otimes \Omega\left(y_{j}\right)^{*} \Omega\left(y_{k}\right)^{*}
$$

using the second relation from Corollary 4.6, which completes the proof.

The polynomial algebra $A=k\left[x_{1}, \ldots, x_{n}\right]$ admits derivations $\partial_{i}=\frac{\partial}{\partial x_{i}}$ defined in the usual way. Similarly its quadratic dual $A^{!}=k\left\langle\Omega\left(y_{1}\right)^{*}, \cdots, \Omega\left(y_{n}\right)^{*}\right\rangle$ has graded derivations $\partial_{i}^{*}$ defined by

$$
\partial_{i}^{*}\left(\Omega(\mathbf{y})^{* \mathbf{b}}\right)=(-1)^{\sum_{j<i} b_{j}} b_{i} \Omega(\mathbf{y})^{* \mathbf{b}-e_{i}}
$$

where $e_{i}$ is the vector with a 1 in position $i$ and zeroes elsewhere and $\Omega(\mathbf{y})^{* \mathbf{b}}$ denotes $\Omega\left(y_{1}\right)^{* b_{1}} \ldots \Omega\left(y_{n}\right)^{* b_{n}}$. We can use these to build a derivation on the Koszul cocomplex:

Lemma 4.9. Let $A=k\left[x_{1}, \ldots, x_{n}\right]$ be a quadratic Poisson algebra and let $A \otimes_{k} A^{!}$ be the Poisson cocomplex computing $\operatorname{HP}^{*}(A)$ with differential $e=e_{P(A)}$. Let $h=$ $\sum_{i} \partial_{i} \otimes \partial_{i}^{*}$.Then

$$
h e+e h=\sum_{i} \partial_{i} H_{i} \otimes 1+1 \otimes \partial_{i}^{*} H_{i}^{*}
$$

where $H_{i}(x)=\left\{x_{i}, x\right\}$ and $H_{i}^{*}(X)=\left\{\Omega\left(y_{i}\right)^{*}, X\right\}=y_{i}^{*} \cdot X$.

Proof. Since $h$ and $e$ are (graded) derivations, so is $h e+e h$. We first show that the right hand side of (4.4) is a derivation.

Since $\{-,-\}$ and its dual are (graded) derivations when one argument is fixed,

$$
\begin{aligned}
& \{x, y\}=\sum_{i} \partial_{i}(x) H_{i}(y)=-\sum_{i} \partial_{i}(y) H_{i}(x) \\
& \{z, w\}=(-1)^{|z|+1} \sum_{i} \partial_{i}^{*}(z) H_{i}^{*}(w)=(-1)^{|z||w|+|w|} \sum_{i} \partial_{i}^{*}(w) H_{i}^{*}(z)
\end{aligned}
$$


for $z, w \in A^{!}$and $x, y \in A$. Furthermore

$$
\partial_{i} H_{i}(x y)=\partial_{i}(x) H_{i}(y)+x \partial_{i} H_{i}(y)+\partial_{i} H_{i}(x) y+H_{i}(x) \partial_{i}(y)
$$

so summing over $i$ and applying (4.5) shows $\sum_{i} \partial_{i} H_{i}$ is a derivation. Similarly so is $\sum_{i} \partial_{i}^{*} H_{i}^{*}$, and thus $\sum_{i} \partial_{i} H_{i} \otimes 1+1 \otimes \partial_{i}^{*} H_{i}^{*}$ is a derivation.

It is easy to see that $h e+e h$ and $\sum_{i} \partial_{i} H_{i} \otimes 1+1 \otimes \partial_{i}^{*} H_{i}^{*}$ agree on $x_{r} \otimes 1$ and $1 \otimes \Omega\left(y_{r}\right)^{*}$ for any $r$. Since these elements generate $A \otimes A^{!}$, the two derivations are equal.

The utility of this result is that in some cases, including that of the semiclassical limits we are interested in, $h e+e h$ acts diagonally on the PBW basis. Therefore some multiple of $h$ will be a contracting homotopy for certain parts of the Koszul cocomplex.

4.3. $q$-deformations of the Koszul cocomplex. Let A be a Koszul $k(q)$-algebra which has a semiclassical limit $A$ such that $\mathrm{A}^{e}$ is a $q$-deformation of $P(A)$ Then $\mathrm{HH}^{*}(\mathrm{~A})$ is computed by the differential graded algebra $\mathrm{A} \otimes \mathrm{A}^{!}$with the differential $e_{\mathrm{A}^{e}}$ and $\mathrm{HP}^{*}(A)$ is computed by $A \otimes A^{!}$with differential $e_{P(A)}$. We want to show that the first of these DGAs is a $q$-deformation of the second.

Proposition 4.10. Let $\mathrm{A}$ be a Koszul $k(q)$-algebra minimally generated by $\mathrm{x}_{1}, \ldots, \mathrm{x}_{n}$ and let $\mathcal{A}$ be the $k\left[q^{ \pm 1}\right]$-form of $\mathrm{A}$ generated by the $\mathrm{x}_{i}$ and admitting a semiclassical limit $A$ which is polynomial on the images of the $\mathrm{x}_{i}$. Suppose the map $P(A) \rightarrow$ $\mathcal{A}^{\prime} /(q-1) \mathcal{A}^{\prime}$ of Lemma 3.6 is an isomorphism. Then the differential graded algebra $\bar{K}_{\mathrm{A}^{e}}(\mathrm{~A})$ is a q-deformation of $\bar{K}_{P(A)}(A)$.

Proof. We split the proof into sections.

Hilbert series. Under our hypotheses,

$$
\operatorname{dim} A_{r}=\operatorname{rank} \mathcal{A}_{r}=\operatorname{dim} \mathrm{A}_{r}
$$

for any $r$. The first equality holds because $\mathcal{A}_{r}$ is free as a $k\left[q^{ \pm 1}\right]$-module, being finitely-generated torsion-free. For the second, if $b_{1}, \ldots$ is a basis of $A_{r}$ then by multiplying by an appropriate scalar we may assume the $b_{i}$ lie in $\mathcal{A}_{r}$. Thus $\operatorname{rank} \mathcal{A}_{r} \geq \operatorname{dim} A_{r}$. Any $k\left[q^{ \pm 1}\right]$-linearly independent set in $\mathcal{A}_{r}$ is $k(q)$-linearly independent in $A_{r}$, so the opposite inequality holds. We get that $A$ has the same Hilbert series as the polynomial algebra $A$. The Hilbert series of a Koszul algebra determines that of its Koszul dual by [13, Corollary 2.2.2], so $A^{!}$has the same Hilbert series as the exterior algebra $A$ ! 
Relations in $\mathbf{A}$ !. Write $\mathrm{A}=T(V) /(R)$ as a quadratic algebra where $V$ has a basis $v_{1}, \ldots, v_{n}$ whose images in $\mathrm{A}$ are $\mathrm{x}_{1}, \ldots, \mathrm{x}_{n}$. Existence of the semiclassical limit implies that for each $i<j$ there is an element $r_{i j}$ of $R$ of the form

$$
v_{i} \otimes v_{j}-v_{j} \otimes v_{i}-(q-1) \hat{\beta}_{i j}
$$

where $\hat{\beta}_{i j}$ lies in the $k\left[q^{ \pm 1}\right]$-span of the $v_{s} \otimes v_{t}$. By substituting $r_{s t}$ into the $\hat{\beta_{i j}}$ we obtain elements $r_{i j}^{\prime}$ of $R$ of the form

$$
v_{i} \otimes v_{j}-v_{j} \otimes v_{i}-(q-1) \hat{\beta}_{i j}^{\prime}+(q-1)^{2} \gamma_{i j}
$$

where only tensors of the form $v_{s} \otimes v_{t}$ for $s<t$ appear in $\beta_{i j}^{\prime}$, and the image of $\hat{\beta}_{i j}^{\prime}$ in $A$ is $\left\{x_{i}, x_{k}\right\}$. These $r_{i j}^{\prime}$ are $k(q)$-linearly independent since they are even linearly independent modulo $q-1$. They form a $k(q)$-basis of $R$, for if $R$ had larger dimension $\operatorname{dim} \mathrm{A}_{2}$ would be smaller than $n(n+1) / 2$.

Let $v_{1}^{*}, \ldots, v_{n}^{*}$ be the basis of $V^{*}$ dual to $v_{1}, \ldots, v_{n}$. For $r<s$ define

$$
\rho_{r s}=v_{r}^{*} \otimes v_{s}^{*}+v_{s}^{*} \otimes v_{r}^{*}-(q-1) \sum_{k<l} \frac{\left\{x_{k}, x_{l}\right\}}{\partial x_{r} \partial x_{s}} v_{l}^{*} \otimes v_{k}^{*}
$$

where $x_{i}$ is the image of $x_{i}$ in $A$. Furthermore define

$$
\rho_{r r}=v_{r}^{*} \otimes v_{r}^{*}-(q-1) \sum_{k<l} \frac{1}{2} \frac{\left\{x_{k}, x_{l}\right\}}{\partial x_{r}^{2}} v_{l}^{*} \otimes v_{k}^{*}
$$

Then for any $r \leq s$ and any $i<j$ we have $\rho_{r s}\left(r_{i j}\right) \in(q-1)^{2} k\left[q^{ \pm 1}\right]$. As before, the $\rho_{r s}$ are linearly independent and there are some elements $\varepsilon_{r s}$ in the $k\left[q^{ \pm 1}\right]$-span of the $v_{i}^{*} \otimes v_{j}^{*}$ such that

$$
\rho_{r s}+(q-1)^{2} \varepsilon_{r s} \quad r \leq s
$$

is a basis of $R^{\perp}$. Recall that $\mathrm{A}^{!}=T\left(V^{*}\right) /\left(R^{\perp}\right)$ and write $\mathrm{x}_{i}^{*}$ for the image of $v_{i}^{*}$ in $\mathrm{A}^{!}$. Let $\mathcal{B}$ be the $k\left[q^{ \pm 1}\right]$-subalgebra of $\mathrm{A}^{!}$generated by the elements $(1-q) \mathrm{x}_{i}^{*}$. The relations (4.6) show that

$$
\mathrm{x}_{i}^{*} \mathrm{x}_{j}^{*}+\mathrm{x}_{j}^{*} \mathrm{x}_{i}^{*}=0 \quad \bmod (q-1)
$$

Since $\mathrm{A}^{!}$and hence $\mathcal{B}$ has the same Hilbert series as $A^{!}$, the quotient $\mathcal{B} /(q-1) \mathcal{B}$ is isomorphic to the exterior algebra $A^{!}$.

Let $\mathcal{B}^{\prime}$ be the subalgebra of $\mathrm{A}^{!} \otimes_{k(q)}\left(\mathrm{A}^{!}\right)^{\text {op }}$ generated by $\mathrm{x}_{i}^{*} \otimes 1+1 \otimes \mathrm{x}_{i}^{*}$ and $(1-q) \otimes \mathrm{x}_{i}^{*}$ for $1 \leq i \leq n$. 
$\mathcal{A}$ is an $\mathcal{A}^{\prime}$-module, $\mathcal{B}$ is a $\mathcal{B}^{\prime}$-module. $\mathrm{A}$ is a $\mathrm{A}^{e}$-module, and we claim that the action of $\mathcal{A}^{\prime} \subseteq \mathrm{A}^{e}$ preserves $\mathcal{A}$. Certainly elements $\mathrm{x}_{i} \otimes 1$ preserve $\mathcal{A}$, and $\left(\mathrm{x}_{i} \otimes 1-1 \otimes \mathrm{x}_{i}\right) \cdot \mathcal{A} \subseteq(q-1) \mathcal{A}$ because of the existence of the semiclassical limit.

Recall that the action of $\mathrm{A}^{!} \otimes_{k(q)}\left(\mathrm{A}^{!}\right)^{\text {op }}$ on $\mathrm{A}^{!}$is

$$
\left(\lambda \otimes \mu^{\prime}\right) \cdot x=(-1)^{|\mu||x|+|\mu|(|\mu|+1) / 2} \lambda x \mu .
$$

Clearly the action of $(1-q) \otimes x_{i}^{*}$ preserves $\mathcal{B}$. The relations (4.6) show that the elements $x_{i}^{*} \otimes 1+1 \otimes x_{i}^{*}$ send generators of $\mathcal{B}$ to $\mathcal{B}$; the general result follows because $\mathrm{x}_{i}^{*} \otimes 1+1 \otimes \mathrm{x}_{i}^{*}$ acts by graded derivations on $\mathcal{B}$.

Let $\mathcal{C}=\mathcal{A} \otimes_{k[q \pm 1]} \mathcal{B}$, and $e_{\mathcal{C}}: \mathcal{C} \rightarrow \mathcal{C}$ be the map induced by left-action of

$\sum_{i}\left(\left(\mathrm{x}_{i} \otimes 1\right) \otimes\left(\mathrm{x}_{i}^{*} \otimes 1+1 \otimes \mathrm{x}_{i}^{*}\right)+\frac{\mathrm{x}_{i} \otimes 1-1 \otimes \mathrm{x}_{i}}{q-1} \otimes\left((1-q) \otimes \mathrm{x}_{i}^{*}\right)\right) \in \mathcal{A}^{\prime} \otimes \mathcal{B}^{\prime}$

This makes it clear that $\mathcal{C}$ with differential $e_{\mathcal{C}}$ is a DGA which is a $k\left[q^{ \pm 1}\right]$-form of $\bar{K}_{\mathrm{A} e}(\mathrm{~A})$. Therefore all we need to complete the proof is:

$\mathcal{C} /(q-1) \mathcal{C}$ is isomorphic as a DGA to $\bar{K}_{P(A)}(A)$. We consider $\bar{K}_{P(A)}(A)$ as the complex $A \otimes_{k} A^{!}$as in the proof of Corollary 4.8.

$\bar{K}_{P(A)}(A)$ is isomorphic as an algebra to $\mathcal{C} /(q-1) \mathcal{C} \cong(\mathcal{A} /(q-1) \mathcal{A}) \otimes$ $(\mathcal{B} /(q-1) \mathcal{B})$. Write $\phi: \bar{K}_{P(A)}(A) \rightarrow(\mathcal{A} /(q-1) \mathcal{A}) \otimes(\mathcal{B} /(q-1) \mathcal{B})$ for the algebra isomorphism that sends $x_{i} \otimes 1$ to the image of $x_{i} \otimes 1$ and $1 \otimes \Omega\left(y_{i}\right)^{*}$ to the image of $(1-q) \otimes x_{i}^{*}$ in $\mathcal{C} /(q-1) \mathcal{C}$. We claim $\phi$ respects the differentials on these DGAs, and since $\bar{K}_{P(A)}(A)$ is generated by the $x_{i} \otimes 1$ and $1 \otimes \Omega\left(y_{i}\right)^{*}$ it is enough to check these elements.

$$
\begin{aligned}
e_{P(A)}\left(x_{i} \otimes 1\right) & =\sum_{j}\left\{x_{j}, x_{i}\right\} \otimes \Omega\left(y_{j}\right)^{*} \\
e_{\mathcal{C}}\left(\mathrm{x}_{i} \otimes 1\right) & =\sum_{j} \beta\left(\mathrm{x}_{j}, \mathrm{x}_{i}\right) \otimes(1-q) \mathrm{x}_{j}^{*}
\end{aligned}
$$

so $\phi\left(e_{P(A)}\left(x_{i} \otimes 1\right)\right)$ equals $e_{\mathcal{C}}\left(\phi\left(x_{i} \otimes 1\right)\right)+(q-1) \mathcal{C}$.

$$
\begin{aligned}
e_{P(A)}\left(1 \otimes \Omega\left(y_{i}\right)^{*}\right) & =-\sum_{j<k} \sum_{r} \frac{\partial\left\{x_{j}, x_{k}\right\}}{\partial x_{i} \partial x_{r}} x_{r} \otimes \Omega\left(y_{j}\right)^{*} \Omega\left(y_{k}\right)^{*} \\
e_{\mathcal{C}}\left(1 \otimes(1-q) \mathrm{x}_{i}^{*}\right) & \equiv \sum_{r} \mathrm{x}_{r} \otimes(1-q)\left(\mathrm{x}_{r}^{*} \mathrm{x}_{i}^{*}+\mathrm{x}_{i}^{*} \mathrm{x}_{r}^{*}\right) \\
& =\sum_{r} \sum_{j<k} \frac{\partial\left\{x_{j}, x_{k}\right\}}{\partial x_{i} \partial x_{r}} \mathrm{x}_{r}^{*} \otimes(1-q)^{2} \mathrm{x}_{k}^{*} \mathrm{x}_{j}^{*}
\end{aligned}
$$

modulo $(q-1) \mathcal{C}$. 
If $\alpha \in k\left[q^{ \pm 1}\right]$ and $M$ is a $k\left[q^{ \pm 1}\right]$-module we say $M$ has $\alpha$-torsion if there is a non-zero element of $M$ annihilated by $\alpha$.

Corollary 4.11. If $H^{*}(\mathcal{C})$ has no $(q-1)$-torsion then $\mathrm{HH}^{*}(\mathrm{~A})$ is a q-deformation of $\operatorname{HP}^{*}(A)$.

Proof. $\mathcal{C} \otimes_{k\left[q^{ \pm 1}\right]} k \cong \bar{K}_{P(A)}(A)$ so $H^{*}\left(\mathcal{C} \otimes_{k\left[q^{ \pm 1}\right]} k\right) \cong \operatorname{HP}^{*}(A)$, and $\mathcal{C} \otimes_{k\left[q^{ \pm 1}\right]}$ $k(q) \cong \bar{K}_{\mathrm{A} e}(\mathrm{~A})$ so $\mathrm{HH}^{*}(\mathrm{~A}) \cong H^{*}\left(\mathcal{C} \otimes_{k\left[q^{ \pm 1}\right]} k(q)\right) \cong H^{*}(\mathcal{C}) \otimes_{k\left[q^{ \pm 1}\right]} k(q)$.

We first show that $\operatorname{HP}^{r}(A)$ and $\operatorname{HH}^{r}(\mathrm{~A})$ have the same Hilbert series for any $r$. The universal coefficient theorem gives an exact sequence of graded modules

$$
0 \rightarrow H^{r j}(\mathcal{C}) \otimes_{k\left[q^{ \pm 1}\right]} k \rightarrow H^{r j}\left(\mathcal{C} \otimes_{k\left[q^{ \pm 1}\right]} k\right) \rightarrow \operatorname{Tor}_{1}^{k\left[q^{ \pm 1}\right]}\left(H^{r+1, j}(\mathcal{C}), k\right) \rightarrow 0
$$

for any $j$. Because $k\left[q^{ \pm 1}\right]$ is a principal ideal domain,

$$
\operatorname{Tor}_{1}^{k\left[q^{ \pm 1}\right]}\left(\frac{k\left[q^{ \pm 1}\right]}{(f)}, k\right)=\operatorname{ker}(k \stackrel{f \cdot}{\rightarrow} k)
$$

and the Tor group in (4.7) vanishes under our hypothesis. So $\operatorname{HP}^{r j}(A) \cong$ $H^{r j}(\mathcal{C}) \otimes_{k\left[q^{ \pm 1}\right]} k$ for any $r, j$, and this has the same dimension as $H^{r j}\left(\mathcal{C} \otimes_{k\left[q^{ \pm 1}\right]}\right.$ $k(q)) \cong \mathrm{HH}^{r j}(\mathrm{~A})$.

Consider the $k\left[q^{ \pm 1}\right]$-form $H^{*}(\mathcal{C}) \otimes_{k\left[q^{ \pm 1}\right]} 1$ of $H^{*}(\mathcal{C}) \otimes_{k\left[q^{ \pm 1}\right]} k(q)$. This is isomorphic as a $k\left[q^{ \pm 1}\right]$-algebra to the quotient of $H^{*}(\mathcal{C})$ by its torsion ideal $T$ (the ideal of all elements annihilated by some $\alpha \in k\left[q^{ \pm 1}\right] \backslash\{0\}$ ). Under our hypothesis $-\otimes_{k\left[q^{ \pm 1}\right]} k$ kills all torsion summands of $H^{*}(\mathcal{C})$, so $H^{*}(\mathcal{C}) \otimes_{k\left[q^{ \pm 1}\right]} \cong$ $\left(H^{*}(\mathcal{C}) / T\right) \otimes_{k\left[q^{ \pm 1}\right]} k$. Thus $\mathrm{HH}^{*}(\mathrm{~A}) \cong H^{*}(\mathcal{C}) \otimes_{k\left[q^{ \pm 1}\right]} k(q)$ is a $q$-deformation of $\operatorname{HP}^{*}(A) \cong H^{*}(\mathcal{C}) \otimes_{k\left[q^{ \pm 1}\right]} k$ via the $k\left[q^{ \pm 1}\right]$-form $H^{*}(\mathcal{C}) \otimes 1 \cong H^{*}(\mathcal{C}) / T$.

In practise the hypothesis of Corollary 4.11 can be checked using the following lemma.

Corollary 4.12. If every element of $\operatorname{ker}\left(e_{\mathcal{C}} \otimes_{k\left[q^{ \pm 1}\right]} k\right)$ lifts to an element of $\operatorname{ker} e_{\mathcal{C}}$, then $H^{*}(\mathcal{C})$ has no $(q-1)$-torsion.

Proof. It is enough to show that $\mathcal{C} / \mathrm{im} e_{\mathcal{C}}$ has no $(q-1)$-torsion under these hypotheses. Since $k\left[q^{ \pm 1}\right]$ is a principal ideal domain, maps between free $k\left[q^{ \pm 1}\right]$ modules can be written in Smith Normal Form. Consequently $\mathcal{C} /$ im $e_{\mathcal{C}}$ has $(q-1)$ torsion if and only if $\operatorname{rank}\left(e_{\mathcal{C}} \otimes k\right)<\operatorname{rank} e_{\mathcal{C}}$ on some graded piece, if and only if the kernel of $e_{\mathcal{C}} \otimes k$ has rank larger than that of $e_{\mathcal{C}}$ on that graded piece. If every element of $\operatorname{ker}\left(e_{\mathcal{C}} \otimes k\right)$ lifts, this cannot happen. 


\section{The quantum plane}

In this section we illustrate some of the results of the previous sections in the case of the quantum plane. The Hochschild cohomology of quantum affine space was described in [15, §3.3] (though it appears difficult to obtain an explicit expression for the $n$th cohomology group), and it is known ([11, Corollary 3.5.2], [12]) that it agrees with the Poisson cohomology of the semiclassical limit.

The coordinate ring A of the quantum plane as defined in Section 3.1 is Koszul since it is a quadratic algebra with a PBW basis of polynomial type. Therefore by Theorem 3.9 and Proposition 4.10, $\bar{K}_{\mathrm{A}} e(\mathrm{~A})$ is a $q$-deformation of $\bar{K}_{P(A)}(A)$, where $A$ is the semiclassical limit of $A$.

The Koszul dual of $A$ is the quantum exterior algebra $A^{!}$, generated by $x^{*}, y^{*}$ subject to $\mathrm{x}^{* 2}=\mathrm{y}^{* 2}=0$ and $q \mathrm{x}^{*} \mathrm{y}^{*}+\mathrm{y}^{*} \mathrm{x}^{*}=0$.

Using this and (4.1) we can compute the Koszul cocomplex $\bar{K}_{\mathrm{A} e}(\mathrm{~A})$. For $m \in \mathbb{Z}$ write $[m]$ for the $q$-integer $\frac{q^{m}-1}{q-1}$. The maps in the Koszul cocomplex are

$$
\begin{aligned}
e_{0}\left(\mathrm{y}^{b} \mathrm{x}^{a}\right) & =[b] \mathrm{x}^{a+1} \mathrm{y}^{n} \otimes \mathrm{x}^{*}-[a] \mathrm{x}^{a} \mathrm{y}^{b+1} \otimes \mathrm{y}^{*} \\
e_{1}\left(\mathrm{y}^{b} \mathrm{x}^{a} \otimes \mathrm{x}^{*}\right) & =[a-1] \mathrm{x}^{a} \mathrm{y}^{b+1} \otimes \mathrm{x}^{*} \mathrm{y}^{*} \\
e_{1}\left(\mathrm{y}^{b} \mathrm{x}^{a} \otimes \mathrm{y}^{*}\right) & =[b-1] \mathrm{x}^{a+1} \mathrm{y}^{b} \otimes \mathrm{x}^{*} \mathrm{y}^{*}
\end{aligned}
$$

so, noting that $\operatorname{gcd}([a],[b])=[\operatorname{gcd}(a, b)]$ in $k\left[q^{ \pm 1}\right]$,

$$
\begin{aligned}
\operatorname{ker} e_{0} & =k\left[q^{ \pm 1}\right] \cdot 1 \\
\left(\operatorname{ker} e_{1}\right)_{n+1} & =k\left[q^{ \pm 1}\right]\left\langle\frac{[b] \mathrm{y}^{b} \mathrm{x}^{n-b} \otimes \mathrm{x}^{*}-[n-b-1] \mathrm{y}^{b+1} \mathrm{x}^{n-1-b} \otimes \mathrm{y}^{*}}{[\operatorname{gcd}(b, n-b-1)]}\right\rangle
\end{aligned}
$$

Therefore $H^{0}(\mathcal{C})=k\left[q^{ \pm 1}\right] \cdot 1$,

$$
\begin{aligned}
& H^{1}(\mathcal{C}) \cong k\left[q^{ \pm 1}\right] \mathrm{x} \otimes \mathrm{x}^{*} \oplus k\left[q^{ \pm 1}\right] \mathrm{y} \otimes \mathrm{y}^{*} \oplus T_{1} \\
& H^{2}(\mathcal{C}) \cong k\left[q^{ \pm 1}\right] \otimes \mathrm{x}^{*} \mathrm{y}^{*} \oplus k\left[q^{ \pm 1}\right] \mathrm{yx} \otimes \mathrm{x}^{*} \mathrm{y}^{*} \oplus T_{2}
\end{aligned}
$$

where $T_{1}$ and $T_{2}$ are direct sums of $k\left[q^{ \pm 1}\right]$-modules of the form $k\left[q^{ \pm 1}\right] /([a])$ for $a \in \mathbb{Z}$. Therefore there is no $(q-1)$-torsion in $H^{*}(\mathcal{C})$, and $\mathrm{HH}(\mathrm{A})$ is a $q$-deformation of $\operatorname{HP}(A)$. Furthermore $\operatorname{HH}(\mathrm{A})$ is one-dimensional in homological degree zero and two-dimensional in degrees 1 and 2.

In fact, for any quantum affine space the two Koszul cocomplexes are actually isomorphic after a change of base field: $\bar{K}_{\mathrm{A} e}(\mathrm{~A}) \cong \bar{K}_{P(A)}(A) \otimes_{k} k(q)$ as cocomplexes. 


\section{6. $2 \times 2$ quantum matrices}

In this section we will compute the Poisson cohomology of $M$, showing that it agrees with the Hochschild cohomology of M. In describing elements of $\bar{K}_{P(M)}(M)$ we write $\Omega_{k l}^{i j}$ for $\Omega(a)^{* i} \Omega(b)^{* j} \Omega(c)^{* k} \Omega(d)^{* l}$ and we omit the $\otimes$ symbol. Similarly in $\bar{K}_{\mathrm{M}}(\mathrm{M})$ we write $\mathrm{f}_{k l}^{i j}$ for $\mathrm{d}^{l} \mathrm{c}^{k} \mathrm{~b}^{j} \mathrm{a}^{i}$ and $\tilde{\Omega}_{k l}^{i j}$ for $\tilde{\Omega}(\mathrm{a})^{* i} \tilde{\Omega}(\mathrm{b})^{* j} \tilde{\Omega}(\mathrm{c})^{* k} \tilde{\Omega}(\mathrm{d})^{* l}$ and omit the $\otimes$ symbol.

We begin by summarizing the results.

Theorem 6.1. $\mathrm{HH}^{*}(\mathrm{M})$ is a q-deformation of $\mathrm{HP}^{*}(M)$. The Poisson centre of $M$ is polynomial in $\Delta=a d-b c$, and for $i \leq 3, \operatorname{HP}^{i}(M)$ is a free $k[\Delta]$-module freely generated by the images of the following elements:

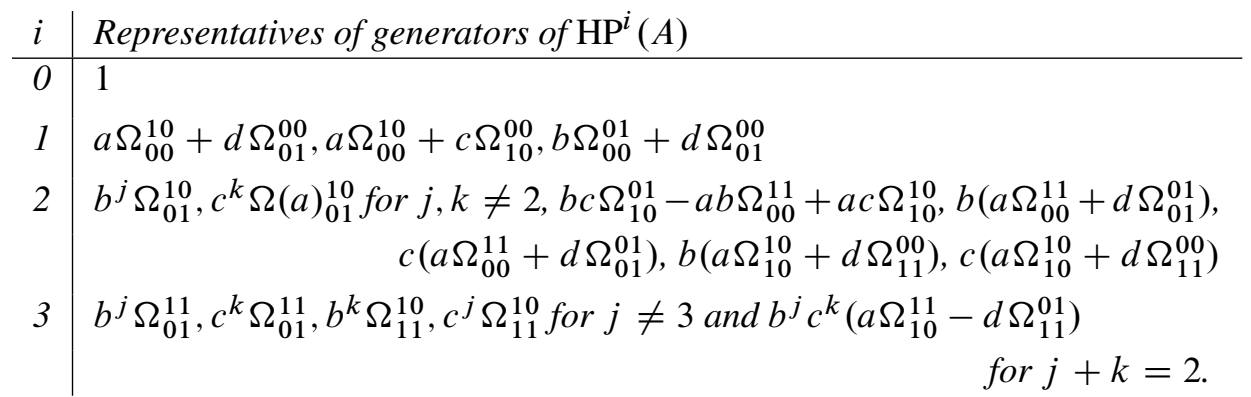

As a $k[\Delta]$-module, $\operatorname{HP}^{4}(M)$ is the direct sum of a trivial module generated by $\Omega_{11}^{11}$ and free summands generated by $b c \Omega_{11}^{11}, b^{j} \Omega_{11}^{11}$ and $c^{k} \Omega_{11}^{11}$ for $j, k>0$.

The theorem is proved as follows. Firstly, $\mathrm{M}$ has a PBW basis of polynomial type, so we may apply Theorem 3.9 and Proposition 4.10 to get that $\bar{K}_{\mathrm{M}^{e}}(\mathrm{M})$ is a $q$-deformation of $\bar{K}_{P(M)}(M)$. We compute $\operatorname{HP}^{*}(M)$ directly using the Koszul cocomplex and show that each cocycle lifts to one for $\bar{K}_{\mathrm{M}}(\mathrm{M})$, so that Corollary 4.12 applies and $\mathrm{HH}^{*}(\mathrm{M})$ is a $q$-deformation of $\mathrm{HP}^{*}(M)$. This is simplified by two observations: first, the boundary of $d^{l} c^{k} b^{j} a^{i} \Omega_{k^{\prime} l^{\prime}}^{i^{\prime} \prime^{\prime}}$ lifts to that of $\mathrm{d}^{l} \mathrm{c}^{k} \mathrm{~b}^{j} \mathrm{a}^{i} \tilde{\Omega}_{k^{\prime} l^{\prime}}^{i^{\prime} j^{\prime}}$ so we only need to lift non-bounding cocycles, and second $\Delta=a d-b c$ lifts to $\Delta_{q}=d a-q^{-1} c b$ so we only need lift a $k[\Delta]$-generating set for the non-bounding cocycles. In practise the lifting is always the most straightforward one possible: $a \Omega_{00}^{10}+b \Omega_{00}^{01}$ lifts to $\mathrm{a} \tilde{\Omega}_{00}^{10}+\mathrm{b} \tilde{\Omega}_{00}^{01}$ and so on.

We need to know the differentials on both Koszul cocomplexes explicitly to check the lifting condition. Since the differential on $\bar{K}_{P(M)}(M)$ is the 'reduction $\bmod q-1$ ' of that for $\bar{K}_{\mathrm{M}^{e}}(\mathrm{M})$, we give the latter in the tables that follow.

$$
\begin{array}{r}
e_{0}\left(\mathrm{f}_{k l}^{i j}\right)=\left([j+k] \mathrm{f}_{k l}^{i+1, j}+q^{-1}[2 l] \mathrm{f}_{k+1, l-1}^{i, j+1}\right) \tilde{\Omega}_{00}^{10}+([l]-[i])\left(\mathrm{f}_{k, l}^{i, j+1} \tilde{\Omega}_{00}^{01}+\mathrm{f}_{k+1, l}^{i j} \tilde{\Omega}_{10}^{00}\right) \\
-\left([j+k] \mathrm{f}_{k, l+1}^{i j}+q^{-1}[2 i] \mathrm{f}_{k+1, l}^{i-1, j+1}\right) \tilde{\Omega}_{01}^{00}
\end{array}
$$




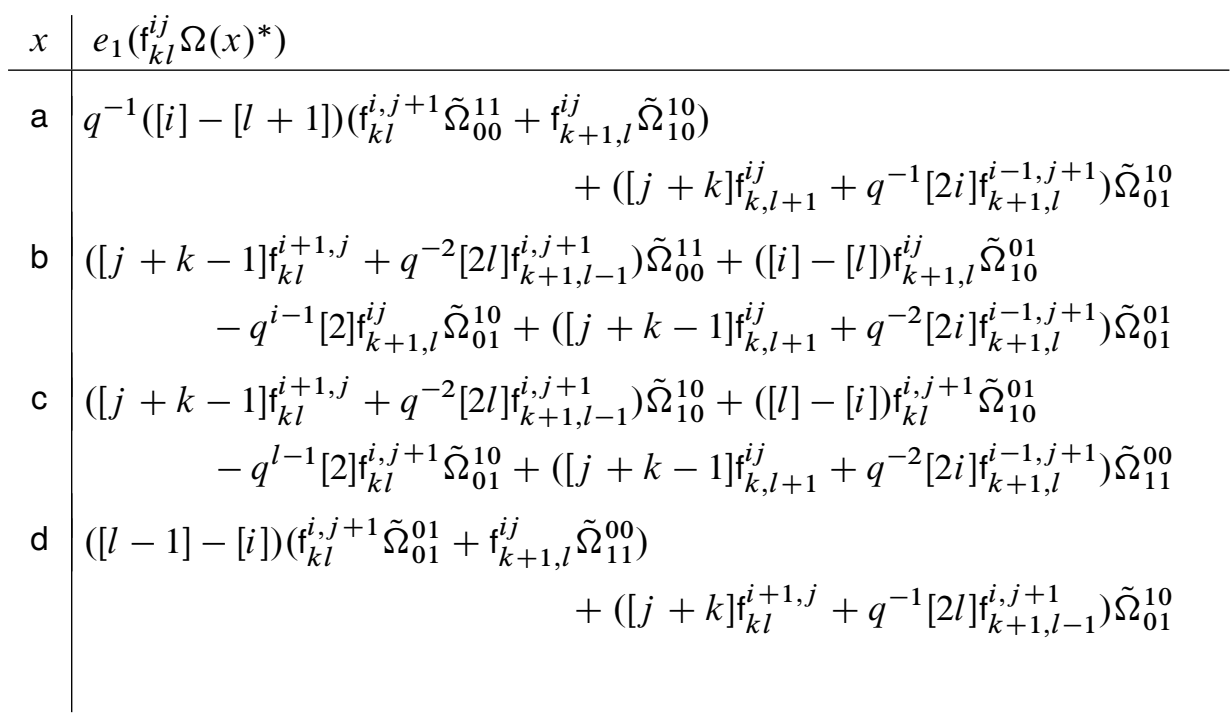

\begin{tabular}{l|l}
$x, y$ & $e_{2}\left(\mathrm{f}_{k l}^{i j} \Omega(x)^{*} \Omega(y)^{*}\right)$ \\
\hline a, b & $q^{-1}([l+1]-[i]) \mathrm{f}_{k+1, l}^{i j} \tilde{\Omega}_{10}^{11}-\left([j+k-1] \mathrm{f}_{k, l+1}^{i j}+q^{-2}[2 i] \mathrm{f}_{k+1, l}^{i-1, j+1}\right) \tilde{\Omega}_{01}^{11}$ \\
a, c & $q^{-1}([i]-[l+1]) \mathrm{f}_{k l}^{i, j+1} \tilde{\Omega}_{10}^{11}-\left([j+k-1] \mathrm{f}_{k, l+1}^{i j}+q^{-2}[2 i] \mathrm{f}_{k+1, l}^{i-1, j+1}\right) \tilde{\Omega}_{11}^{10}$ \\
a, d & $q^{-1}([i]-[l])\left(\mathrm{f}_{k l}^{i, j+1} \tilde{\Omega}_{01}^{11}+\mathrm{f}_{k+1, l}^{i j} \tilde{\Omega}_{11}^{10}\right)$ \\
b, c & $\left([j+k-2] \mathrm{f}_{k l}^{i+1, j}+q^{-3}[2 l] \mathrm{f}_{k+1, l-1}^{i, j+1}\right) \tilde{\Omega}_{10}^{11}-q^{l-2}[2] \mathrm{f}_{k l}^{i, j+1} \tilde{\Omega}_{01}^{11}$ \\
$\quad+q^{i-2}[2] \mathrm{f}_{k+1, l}^{i j} \tilde{\Omega}_{11}^{10}-\left([j+k-2] \mathrm{f}_{k, l+1}^{i j}+q^{-3}[2 i] \mathrm{f}_{k+1, l}^{i-1, j+1}\right) \tilde{\Omega}_{11}^{01}$ \\
b, d & $\left([j+k-1] \mathrm{f}_{k l}^{i+1, j}+q^{-2}[2 l] \mathrm{f}_{k+1, l-1}^{i, j+1}\right) \tilde{\Omega}_{01}^{11}+([i]-[l-1]) \mathrm{f}_{k+1, l}^{i j} \tilde{\Omega}_{11}^{01}$ \\
c, d & $\left([j+k-1] \mathrm{f}_{k l}^{i+1, j}+q^{-2}[2 l] \mathrm{f}_{k+1, l-1}^{i, j+1}\right) \tilde{\Omega}_{11}^{10}+([l-1]-[i]) \mathrm{f}_{k l}^{i, j+1} \tilde{\Omega}_{11}^{01}$
\end{tabular}

\begin{tabular}{r|l}
$i^{\prime}, j^{\prime}, k^{\prime}, l^{\prime}$ & $e_{3}\left(\mathrm{f}_{k l}^{i j} \tilde{\Omega}_{k^{\prime} l^{\prime}}^{i^{\prime} j^{\prime}}\right)$ \\
\hline $1,1,1,0$ & {$[j+k-2] \mathrm{f}_{k, l+1}^{i j} \tilde{\Omega}_{11}^{11}+q^{-3}[2 i] \mathrm{f}_{k+1, l}^{i-1, j+1} \tilde{\Omega}_{11}^{11}$} \\
$1,1,0,1$ & $q^{-1}([l]-[i]) \mathrm{f}_{k+1, l}^{i j} \tilde{\Omega}_{11}^{11}$ \\
$1,0,1,1$ & $q^{-1}([i]-[l]) \mathrm{f}_{k l}^{i, j+1} \tilde{\Omega}_{11}^{11}$ \\
$0,1,1,1$ & {$[j+k-2] \mathrm{f}_{k l}^{i+1, j} \tilde{\Omega}_{11}^{11}+q^{-3}[2 l] \mathrm{f}_{k+1, l-1}^{i, j+1} \tilde{\Omega}_{11}^{11}$}
\end{tabular}


The formulas are obtained by computing directly using the definition of the Koszul cocomplex and the presentations of $M$ and $M !$ given in (3.4) and Example 4.1.

We want to find a contracting homotopy to show that certain portions of $\bar{K}_{P(M)}(M)$ are exact. To this end we define another grading on $\bar{K}_{P(M)}(M)$ :

Definition 6.2. The grade of $a^{i} b^{j} c^{k} d^{l} \Omega_{k^{\prime}, l^{\prime}}^{i^{\prime}, j^{\prime}} \in \bar{K}_{P(M)}(M)$ is $i-i^{\prime}-l+l^{\prime}$.

The differential $e_{P(M)}$ preserves grade, as is easily seen from the tables above.

Lemma 6.3. $(h e+e h)\left(f_{k l}^{i j} \Omega_{k^{\prime} l^{\prime}}^{i^{\prime} j^{\prime}}\right)=2\left(l-l^{\prime}-i+i^{\prime}\right) f_{k l}^{i j} \Omega_{k^{\prime} l^{\prime}}^{i^{\prime} j^{\prime}}$

Proof. This is an application of Lemma 4.9. Computing using the bracket formulas (3.5) shows

$$
\left(\partial_{a} H_{a}+\partial_{b} H_{b}+\partial_{c} H_{c}+\partial_{d} H_{d}\right) f=2(l-i) f_{k l}^{i j} .
$$

Next, the second relation of Corollary 4.6 gives the following description of the action of $P(M)^{!}$on $M^{!}$(with a slight abuse of notation):

$$
\begin{aligned}
a^{*} \cdot \Omega(b)^{*} & =b^{*} \cdot \Omega(a)^{*}=-\Omega(a)^{*} \Omega(b)^{*} \\
a^{*} \cdot \Omega(c)^{*} & =c^{*} \cdot \Omega(a)^{*}=-\Omega(a)^{*} \Omega(c)^{*} \\
a^{*} \cdot \Omega(d)^{*} & =d^{*} \cdot \Omega(a)^{*}=0 \\
b^{*} \cdot \Omega(c)^{*} & =c^{*} \cdot \Omega(b)^{*}=-2 \Omega(a)^{*} \Omega(d)^{*} \\
b^{*} \cdot \Omega(d)^{*} & =d^{*} \cdot \Omega(b)^{*}=0 \\
c^{*} \cdot \Omega(d)^{*} & =d^{*} \cdot \Omega(c)^{*}=-\Omega(c)^{*} \Omega(d)^{*}
\end{aligned}
$$

and that $x^{*} \cdot \Omega(x)^{*}=0$ for $x=a, b, c, d$. From these, a tedious calculation shows that

$$
\left(\partial_{a}^{*} H_{a}^{*}+\partial_{b}^{*} H_{b}^{*}+\partial_{c}^{*} H_{c}^{*}+\partial_{d}^{*} H_{d}^{*}\right) \Omega_{k^{\prime} l^{\prime}}^{i^{\prime} j^{\prime}}=2\left(i^{\prime}-l^{\prime}\right) \Omega_{k^{\prime} l^{\prime}}^{i^{\prime} j^{\prime}}
$$

Putting these together gives the formula claimed.

This means that a suitable scalar multiple of $h$ is a contracting homotopy on any summand of $\bar{K}_{P(M)}(M)$ with nonzero grade. Write $K_{0}(M)$ for the subcomplex of $\bar{K}_{P(M)}(M)$ consisting of all elements of grade zero, and let $E_{n}$ be the induced differential in homological degree $n$. Then $\operatorname{HP}^{*}(M) \cong H^{*}\left(K_{0}(M), E\right)$, so we work only with the cocomplex $K_{0}(M)$.

\subsection{Computations.}

Lemma 6.4. $\operatorname{HP}^{0}(M)=k[\Delta]$, and $\Delta$ lifts to $\Delta_{q}=d a-q^{-1} c b$.

Proof. $K_{0}(M)_{0}$ consists of elements of the form $\sum \lambda_{i j k}(a d)^{i} b^{j} c^{k}$, and such an element is in ker $E_{0}$ if and only if it has trivial bracket with $a$, if and only if

$$
2 i \lambda_{i, j-1, k-1}=-(j+k) \lambda_{i-1, j, k}
$$


for all $i, j, k$, where $\lambda_{i j k}$ should be interpreted as zero if any of its indices are negative. If $\lambda_{i j k}$ is a solution of this recurrence and $j>k$ then $\lambda_{i j k}=0$ : if $k$ is minimal such that there exists $j>k$ with $\lambda_{i j k} \neq 0$ then $(j+k) \lambda_{i j k}=$ $-2(i+1) \lambda_{i+1, j-1, k-1}=0$ contradicting $\lambda_{i j k} \neq 0$. By symmetry $\lambda_{i j k}=0$ if $j \neq k$.

Writing $\lambda_{i j}$ for $\lambda_{i j j}$ gives

$$
i \lambda_{i, j-1}=-j \lambda_{i-1, j}
$$

For fixed $i+j$, any solution is a scalar multiple of $\lambda_{i j}=(-1)^{i}\left(\begin{array}{c}i+j \\ i\end{array}\right)$, thus $\sum \lambda_{i j}(a d)^{i}(b c)^{j}$ is a polynomial in $\Delta$. The claim about lifting is easily verified.

Therefore $E_{0}$ is a $k[\Delta]$-map with kernel equal to $k[\Delta]$. As a $k[\Delta]$-module, $K_{0}(M)=\left\langle(a d)^{i} b^{j} c^{k}: i, j, k \geq 0\right\rangle$ is free on the generators $b^{j} c^{k}$, so im $E_{0}$ is free on the image of $b^{j} c^{k}$ for $j, k$ not both zero. Using the description of the differential above, we get:

Lemma 6.5. im $E_{0}$ is freely generated by $b^{j} c^{k}\left(a \Omega_{00}^{10}-d \Omega_{01}^{00}\right)$ for $j, k$ not both zero.

In the above Lemma and from now on, the terms 'free generators' and 'freely generated' refer to the $k[\Delta]$-module structure.

Lemma 6.6. ker $E_{1}$ is freely generated by $b \Omega_{00}^{01}+a \Omega_{00}^{10}, a \Omega_{00}^{10}+c \Omega_{10}^{00}$ and all $b^{j} c^{k}\left(a \Omega_{00}^{10}-d \Omega_{01}^{00}\right)$ with $j, k \geq 0$.

Proof. $K_{0}(M)_{1}$ is spanned by elements of the form $(a d)^{i} a b^{j} c^{k} \Omega_{00}^{10},(a d)^{i} b^{j} c^{k} \Omega_{00}^{01}$, $(a d)^{i} b^{j} c^{k} \Omega_{10}^{00},(a d)^{i} d b^{j} c^{k} \Omega_{01}^{00}$ and is therefore freely generated by the elements $a b^{j} c^{k} \Omega_{00}^{10}, b^{j} c^{k} \Omega_{00}^{01}, b^{j} c^{k} \Omega_{10}^{00}, d b^{j} c^{k} \Omega_{01}^{00}$. The images under $E_{1}$ of these free generators are

$$
\begin{aligned}
E_{1} a b^{j} c^{k} \Omega_{00}^{10}=E_{1} d b^{j} c^{k} \Omega_{01}^{00} & =\left((j+k+2) b^{j+1} c^{j+1}+(j+k) \Delta b^{j} c^{k}\right) \Omega_{01}^{10} \\
E_{1} b^{j} c^{k} \Omega_{00}^{01} & =(j+k-1) b^{j} c^{k}\left(a \Omega_{00}^{11}+d \Omega_{01}^{01}\right)-2 b^{j} c^{k+1} \Omega_{01}^{10} \\
E_{1} b^{j} c^{k} \Omega_{10}^{00} & =(j+k-1) b^{j} c^{k}\left(a \Omega_{10}^{10}+d \Omega_{11}^{00}\right)-2 b^{j+1} c^{k} \Omega_{01}^{10}
\end{aligned}
$$

Therefore if $p_{j k}, q_{j k}, r_{j k}, s_{j k}$ are polynomials, the image of

$$
\sum p_{j k}(\Delta) a b^{j} c^{k} \Omega_{00}^{10}+q_{j k}(\Delta) b^{j} c^{k} \Omega_{00}^{01}+r_{j k}(\Delta) b^{j} c^{k} \Omega_{10}^{00}+s_{j k}(\Delta) d b^{j} c^{k} \Omega_{01}^{00}
$$

is

$$
\begin{aligned}
& \sum(j+k-1) q_{j k}(\Delta)\left(a b^{j} c^{k} \Omega_{00}^{11}+d b^{j} c^{k} \Omega_{01}^{01}\right) \\
& +\sum(j+k-1) r_{j k}(\Delta)\left(a b^{j} c^{k} \Omega_{10}^{10}+d b^{j} c^{k} \Omega_{11}^{00}\right) \\
& +\sum\left(p_{j k}(\Delta)+s_{j k}(\Delta)\right)\left((j+k+2) b^{j+1} c^{k+1}+(j+k) \Delta b^{j} c^{k}\right) \Omega_{01}^{10} \\
& -\sum\left(2 q_{j k}(\Delta) b^{j} c^{k+1}+2 r_{j k}(\Delta) b^{j+1} c^{k}\right) \Omega_{01}^{10} .
\end{aligned}
$$


The terms $a b^{j} c^{k} \Omega_{10}^{10}$ are free generators of the $\Omega_{10}^{10}$ component of $K_{0}(M)_{2}$, and so the only way for the $\Omega_{10}^{10}$ component of this expression to vanish is if $r_{j k}=0$ for $j+k \neq 1$. Similarly we must have $q_{j k}=0$ for $j+k \neq 1$.

The coefficient of $c^{2} \Omega_{01}^{10}$ in this sum is $2 q_{01}(\Delta) c^{2} \Omega_{01}^{10}$, therefore $q_{01}=0$, and similarly $r_{10}=0$. The condition for (6.1) to be in $\operatorname{ker} E_{1}$ is therefore that $r_{j k}=$ $q_{j k}=q_{01}=r_{10}$ for $j+k \neq 1$ and

$$
\begin{aligned}
-q_{10}-r_{01}+p_{00}+s_{00} & =0 \\
p_{j k}+s_{j k} & =0
\end{aligned}
$$

for $j, k$ not both zero. This is equivalent to our claim about the kernel generators.

We say an element $\sum \alpha_{i j k l}^{i^{\prime} j^{\prime} k^{\prime} l^{\prime}} f_{k l}^{i j} \Omega_{k^{\prime} l^{\prime}}^{i^{\prime} j^{\prime}}$ of ker $E_{*}$ lifts trivially if $\sum \alpha_{i j k l}^{i^{\prime} j^{\prime} k^{\prime} l^{\prime}} \mathrm{f}_{k l}^{i j} \tilde{\Omega}_{k^{\prime} l^{\prime}}^{i^{\prime} j^{\prime}}$ is a cocycle for $\bar{K}_{\mathrm{M}}(\mathrm{M})$.

Corollary 6.7. $\mathrm{HP}^{1}(M)$ is freely generated by the images of $b \Omega_{00}^{01}+a \Omega_{00}^{10}, a \Omega_{00}^{10}+$ $c \Omega_{10}^{00}$ and $a \Omega_{00}^{10}-d \Omega_{01}^{00}$. Each of these elements lifts trivially to an element of $\mathrm{HH}^{1}(\mathrm{M})$.

Proof. The first part follows immediately from the previous two lemmas. The second follows from the description of the differential in $\bar{K}_{\mathrm{M}}(\mathrm{M})$ given earlier.

Lemma 6.8. im $E_{1}$ is freely generated by

$$
c^{2} \Omega_{01}^{10}, \quad b^{2} \Omega_{01}^{10}, \quad\left(b^{j+1} c^{k+1}+\frac{j+k}{j+k+2} \Delta b^{j} c^{k}\right) \Omega_{01}^{10}
$$

for $j, k \geq 0$ and for $j+k \neq 1$,

$$
\begin{aligned}
& b^{j} c^{k}\left(a \Omega_{00}^{11}+d \Omega_{01}^{01}\right)-\frac{2}{j+k-1} \Omega_{01}^{10} \\
& b^{j} c^{k}\left(a \Omega_{10}^{10}+d \Omega_{11}^{00}\right)-\frac{2}{j+k-1} \Omega_{01}^{10} .
\end{aligned}
$$

Proof. From our description of ker $E_{1}$ it follows that im $E_{1}$ is freely generated by the images of $a b^{j} c^{k} \Omega_{00}^{10}$ for any $j, k, b^{j} c^{k} \Omega_{00}^{01}$ and $b^{j} c^{k} \Omega_{10}^{00}$ for $j+k \neq 1$ and $c \Omega_{00}^{01}$ and $b \Omega_{10}^{00}$. These images are non-zero scalar multiples of the given elements.

Lemma 6.9. $\operatorname{ker} E_{2}$ is freely generated by

$$
b^{k} c^{k}\left(a \Omega_{00}^{11}+d \Omega_{01}^{01}\right), \quad b^{j} c^{k}\left(a \Omega_{10}^{10}+d \Omega_{11}^{00}\right), \quad b^{j} c^{k} \Omega_{01}^{10}
$$

for $j, k \geq 0$ and $b c \Omega_{10}^{01}-a b \Omega_{00}^{11}+a c \Omega_{10}^{10}$. 
Proof. $E_{2}$ kills $b^{j} c^{k} \Omega_{01}^{10}$ and acts on the other free generators of $K_{0}(M)_{2}$ as follows:

$$
\begin{aligned}
E_{2} a b^{j} c^{k} \Omega_{00}^{11} & =-E_{2} d b^{j} c^{k} \Omega_{01}^{01} \\
& =\left((j+k+1) b^{k+1} c^{k+1}+(j+k-1) \Delta b^{j} c^{k}\right) \Omega_{01}^{11} \\
E_{2} a b^{j} c^{k} \Omega_{10}^{10} & =-E_{2} d b^{j} c^{k} \Omega_{11}^{00} \\
& =\left((j+k+1) b^{k+1} c^{k+1}+(j+k-1) \Delta b^{j} c^{k}\right) \Omega_{11}^{10} \\
E_{2} b^{j} c^{k} \Omega_{10}^{01} & =(j+k-2) b^{j} c^{k}\left(a \Omega_{10}^{11}-d \Omega_{11}^{01}\right)+2 b^{j} c^{k}\left(c \Omega_{11}^{10}-b \Omega_{01}^{11}\right)
\end{aligned}
$$

As in the calculation of ker $E_{1}$ we see by considering the $\Omega_{10}^{11}$ term that a kernel element of the form

$$
\begin{aligned}
\sum p_{j k}(\Delta) a b^{j} c^{k} \Omega_{00}^{11}+q_{j k}(\Delta) a b^{j} c^{k} \Omega_{10}^{10}+r_{j k}(\Delta) b^{j} c^{k} \Omega_{10}^{01} & \\
& +s_{j k}(\Delta) d b^{j} c^{k} \Omega_{01}^{01}+t_{j k}(\Delta) d b^{j} c^{k} \Omega_{11}^{00}
\end{aligned}
$$

must have $r_{j k}=0$ unless $j+k=2$, and since the $b^{3} \Omega_{01}^{11}$ and $c^{3} \Omega_{11}^{10}$ coefficients must vanish $r_{j k}=0$ unless $j=k=1$. The condition for an element obeying these restrictions on $r_{j k}$ to lie in the kernel is that

$$
\begin{aligned}
r_{11}+q_{01}-t_{01} & =0 & -r_{11}+p_{10}-s_{10} & =0 \\
p_{j k}-s_{j k} & =0 & q_{l m}-r_{l m} & =0
\end{aligned}
$$

for $(j, k) \neq(1,0)$ and $(l, m) \neq(0,1)$. Therefore the kernel is as claimed.

Corollary 6.10. $\operatorname{HP}^{2}(M)$ is freely generated by the images of

$$
\begin{gathered}
b\left(a \Omega_{00}^{11}+d \Omega_{01}^{01}\right), \quad b\left(a \Omega_{10}^{10}+d \Omega_{11}^{00}\right), \quad c\left(a \Omega_{00}^{11}+d \Omega_{01}^{01}\right), \quad c\left(a \Omega_{10}^{10}+d \Omega_{11}^{00}\right), \\
b c \Omega_{10}^{01}-a b \Omega_{00}^{11}+a c \Omega_{10}^{10}, \quad b^{r} \Omega_{01}^{10}, \quad c^{r} \Omega_{01}^{10}
\end{gathered}
$$

for $r \neq 2$. Each of these elements lifts trivially to an element of $\mathrm{HH}^{2}(\mathrm{M})$.

Proof. The first statement follows from our calculation of $\operatorname{ker} E_{2}$ and $\operatorname{im} E_{1}$, the second by computing using the description of the differential on $\bar{K}_{\mathrm{M}}(\mathrm{M})$ given earlier.

Lemma 6.11. im $E_{2}$ is freely generated by

$$
\left(b^{j+1} c^{k+1}+\frac{j+k-1}{j+k+1} \Delta b^{j} c^{k}\right) \Omega_{01}^{11}, \quad\left(b^{j+1} c^{k+1}+\frac{j+k-1}{j+k+1} \Delta b^{j} c^{k}\right) \Omega_{11}^{10}
$$

for any $j, k \geq 0$,

$$
b^{j} c^{k}\left(a \Omega_{10}^{11}-d \Omega_{11}^{01}\right)+2 b^{j} c^{k}\left(c \Omega_{11}^{10}-b \Omega_{01}^{11}\right)
$$

for $j+k \neq 2, b^{3} \Omega_{01}^{11}$ and $c^{3} \Omega_{11}^{10}$. 
Proof. The computation of $\operatorname{ker} E_{2}$ shows that im $E_{2}$ is free on the images of $b^{j} c^{k} a \Omega_{00}^{11}, b^{j} c^{k} a \Omega_{10}^{10}, b^{j} c^{k} \Omega_{10}^{01}$ for $j+k \neq 2, b^{2} \Omega_{01}^{01}+a b \Omega_{10}^{10}$ and $c^{2} \Omega_{10}^{01}-a c \Omega_{00}^{11}$ which are, up to a scalar multiple, the given generators.

Lemma 6.12. $\operatorname{ker} E_{3}$ is freely generated by

$$
b^{j} c^{k} \Omega_{11}^{10}, b^{j} c^{k} \Omega_{01}^{11}, \text { and } b^{j} c^{k}\left(a \Omega_{10}^{11}-d \Omega_{11}^{01}\right)
$$

for all $j, k \geq 0$.

Proof. $E_{3}$ kills $b^{j} c^{k} \Omega_{11}^{10}$ and $b^{j} c^{k} \Omega_{01}^{11}$ and sends $b^{j} c^{k} a \Omega_{10}^{11}$ and $b^{j} c^{k} d \Omega_{11}^{01}$ to $\left((j+k) b^{j+1} c^{k+1}+(j+k-2) \Delta b^{j} c^{k}\right) \Omega_{11}^{11}$. The result follows immediately.

Again we can read off the cohomology group:

Corollary 6.13. $\operatorname{HP}^{3}(M)$ is freely generated by the images of

$$
b^{j} \Omega_{01}^{11}, \quad c^{k} \Omega_{01}^{11}, \quad b^{k} \Omega_{11}^{10}, \quad c^{j} \Omega_{11}^{10}
$$

for $j \neq 3$ together with ax $\Omega_{10}^{11}-d x \Omega_{11}^{01}$ for $x=b^{2}, b c, c^{2}$. Each of these elements lifts trivially to an element of $\mathrm{HH}^{3}(\mathrm{M})$.

Lemma 6.14. im $E_{3}$ is freely generated by $\Delta \Omega_{11}^{11}$ and $\left(b^{j+1} c^{k+1}+\frac{j+k-2}{j+k} b^{j} c^{k}\right) \Omega_{11}^{11}$ for $j, k$ not both zero.

Proof. Our computation of $\operatorname{ker} E_{3}$ shows that $\operatorname{im} E_{3}$ is free on the images of $b^{j} c^{k} a \Omega_{10}^{11}$, which up to a nonzero scalar multiple are the generators given.

Corollary 6.15. $\operatorname{HP}^{4}(M)$ is generated as a $k[\Delta]$-module by $\Omega_{11}^{11}, b^{j} \Omega_{11}^{11}$ and $c^{j} \Omega_{11}^{11}$ for $j, k \geq 0 . k[\Delta]$ acts trivially on $\Omega_{11}^{11}$ and freely on the other generators.

Since $E_{4}=0$, all kernel elements lift trivially.

\section{Acknowledgements}

I would like to thank Stéphane Launois for proposing this problem to me and for many helpful suggestions. This research was financially supported through his EPSRC First Grant EP/I018549/1.

\section{References}

[1] R.-O. Buchweitz, E. L. Green, D. Madsen and Ø. Solberg. Finite Hochschild cohomology without finite global dimension. Math. Res. Lett., 12 (2005) no.56, 805-816. Zbl 1138.16003 MR 2189240 
[2] R.-O. Buchweitz, E. L. Green, N. Snashall and Ø. Solberg. Multiplicative structures for Koszul algebras. Q. J. Math., 59 (2008) no. 4, 441-454. Zbl 1191.16027 MR 2461267

[3] H. Cartan and S. Eilenberg. Homological algebra. Princeton Landmarks in Mathematics. Princeton University Press, Princeton, NJ, 1999. With an appendix by David A. Buchsbaum, Reprint of the 1956 original. Zbl 0933.18001 MR 1731415

[4] F. Dumas. Rational equivalence for poisson polynomial algebras. Lecture notes, December 2011.

[5] P. Feng and B. Tsygan. Hochschild and cyclic homology of quantum groups. Comm. Math. Phys., 140 (1991) no. 3, 481-521. Zbl 0743.17020 MR 1130695

[6] E. L. Green, G. Hartman, E. N. Marcos and Ø. Solberg. Resolutions over Koszul algebras. Arch. Math. (Basel), 85 (2005) no. 2, 118-127. Zbl 1096.16011 MR 2161801

[7] K. R. Goodearl. Semiclassical limits of quantized coordinate rings in Advances in ring theory, Trends Math., pages 165-204. Birkhäuser/Springer Basel AG, Basel, 2010. Zbl 1202.16027 MR 2664671

[8] J. Huebschmann. Poisson cohomology and quantization. J. Reine Angew. Math., 408 (1990) 57-113. Zbl 0699.53037 MR 1058984

[9] C. Kassel. L'homologie cyclique des algébres enveloppantes. Invent. Math., 91 (1988) no. 2, 221-251. Zbl 0653.17007 MR 922799

[10] S. Launois and T. H. Lenagan. The first Hochschild cohomology group of quantum matrices and the quantum special linear group. J. Noncommut. Geom., 1 (2007) no. 3, 281-309. Zbl 1137.16015 MR 2314098

[11] S. Launois and L. Richard. Twisted Poincaré duality for some quadratic Poisson algebras. Lett. Math. Phys., 79, no. 2, 161-174. Zbl 1139.17008 MR 2301394

[12] P. Monnier. Formal Poisson cohomology of quadratic Poisson structures. Lett. Math. Phys., 59 (2002) no. 3, 253-267. Zbl 1010.53059 MR 1904986

[13] A. Polishchuk and L. Positselski. Quadratic algebras, volume 37 of University Lecture Series. American Mathematical Society, Providence, RI, 2005. Zbl 1145.16009 MR 2177131

[14] S. B. Priddy. Koszul resolutions. Trans. Amer. Math. Soc., 152 (1970) 39-60. Zbl 0261.18016 MR 265437

[15] A. Sitarz. Twisted Hochschild homology of quantum hyperplanes. K-Theory, 35 (2005) no. 1-2, 187-198. Zbl 1110.46046 MR 2240220

[16] M. van den Bergh. Noncommutative homology of some three-dimensional quantum spaces in Proceedings of Conference on Algebraic Geometry and Ring Theory in honor of Michael Artin, Part III (Antwerp, 1992), volume 8, pages 213-230, 1994. Zbl 0814.16006 MR 1291019 
[17] M. Wambst. Complexes de Koszul quantiques. Ann. Inst. Fourier (Grenoble), 43 (1993) no. 4, 1089-1156. Zbl 0810.16010 MR 1252939

[18] C. A. Weibel. An introduction to homological algebra, volume 38 of Cambridge Studies in Advanced Mathematics. Cambridge University Press, Cambridge, 1994. Zbl 0797.18001 MR 1269324

Received 28 June, 2013; revised 09 January, 2014

M. Towers, School of Mathematics, Statistics and Actuarial Science, University of Kent, Cornwallis Building, Canterbury CT2 7NF, UK

E-mail: m.towers@kent.ac.uk 